\title{
Effect of a poloidal electric field on neoclassical transport in a multispecies tokamak plasma
}

\author{
K. Indireshkumar and W. M. Stacey, Jr. \\ Fusion Research Center and \\ Nuclear Engineering Program \\ Georgia Institute of Technology \\ Atlanta, GA 30332
}

December 1992

\section{DISCLAIMER}

This report was prepared as an account of work sponsored by an agency of the United States Government. Neither the United States Government nor any agency thereof, nor any of their employees, makes any warranty, express or implied, o: assumes any legal liability or responsibility for the accuracy, completeness, or usefulness of any information, apparatus, product, or process discloeed, or represents that its use would not infringe privately owned rights. Reference herein to any specific commercial product, process, or service by trade name, trademark, manufacturer, or otherwise does not necesearily constitute or imply its endorsement, recommendation, or favoring by the United States Government or any agency thereof. The views and opinions of authors expressed herein do not necessarily state or reflect those of the United States Government or any agency thereof.

\section{MASTER}




\begin{abstract}
The effects of a poloidal potential variation of order $\epsilon$, which is likely to be produced during high power cyclotron wave heating or neutral beam injection, upon neoclassical particle transport and plasma current are studied theoretically, for a realistic tokamak plasma with significant impurity content. Using an approximate collision operator, an analytic procedure is employed to calculate the transport coefficients in the low collisionality regime for a large aspect ratio tokamak. In the presence of carbon impurity, the ion diffusion coefficients are generally found to increase by a factor of $\sim$ 2. Inclusion of the effects of a poloidal electric field is found to result in an increase in the bootstrap current if the potential on the outside of the tokamak is greater than that on the inside (as during ICRH or NBI) and the density profiles are more peaked than roughly the square root of the temperature profiles.
\end{abstract}




\section{INTRODUCTION}

High power ion and electron cyclotron wave heating (ICRH and ECRH) can give rise to significant in-out poloidal asymmetry in the electrostatic potential. It has been shown ${ }^{1}$ that, in the low collisionality regime, cyclotron wave heating causes increased trapping, leading to a poloidal variation in the potential, which can reach magnitudes of the order $\epsilon$, i.e., $e \bar{\Phi}(\theta) / T \sim O(\epsilon)$. Here, $e$ is the unit charge, $\tilde{\Phi}(\theta)$ is the poloidally varying part of the potential, $T$ is the typical temperature of the plasma constituents, and $\epsilon=r / R$ where $r$ and $R$ are the minor and major radii of the tokamak respectively. It has been suggested ${ }^{2}$ that both parallel and perpendicular neutral beam injection heating (NBI) can also give rise to a significant poloidal electric field.

A poloidal potential variation of order $\epsilon$ can cause a significant change in the neoclassical transport coefficients in the banana regime. It has been shown by Chang ${ }^{2}$ and Shurygin $e t$ al. ${ }^{3}$ that an in-out potential asymmetry of order $\epsilon$ can cause a significant enhancement of the neoclassical transport coefficients in a simple (electron-ion) plasma.

In a realistic scenario, the plasma is contaminated by impurities. It is well known ${ }^{4}$ that the presence of an impurity, even in small amounts, can cause a qualitative and quantitative change in the neoclassical transport. Indeed, in an impure plasma, ion diffusion exceeds electron diffusion by approximately the square root of the ion to electron mass ratio. Hence, it is important to investigate the effect of an externally induced poloidal electric field on neoclassical transport in an impure plasma in the banana regime, which is the purpose of this paper.

We will be primarily concerned with calculation of the particle transport and plasma current in the low collisionality, or banana, regime. Furthermore, we limit our consideration to a large aspect ratio $(\epsilon \ll 1)$ tokamak. In an impure plasma, the presence of a poloidal electric field affects the collisional coupling between the main ions and the impurity ions in addition to affecting the electron-ion collisional coupling. The result of such a modification is an enhancement (or reduction) of the ion transport as well as plasma current.

The paper is organized as follows. In Sec. II, we examine particle trapping in the 
presence of a poloidal electric field. We solve the drift kinetic equation in Sec. III, using an approximate collision operator developed by Hirshman et al. ${ }^{5}$ The solution methodology closely parallels the analytical technique in Ref. 5 . We end this section by deriving a set of equations for the so called restoring coefficients. ${ }^{5}$ In Sec. IV, the restoring coefficients are used to obtain the electron and ion transport coefficients. In Sec. V, we consider modifications to the plasma current due to the poloidal electric field. Section VI contains a summary and concluding remarks.

\section{EFFECT OF POLOIDAL ELECTRIC FIELD ON PARTICLE TRAPPING}

The presence of a poloidal potential variation of order $\epsilon$ significantly alters the nature of particle trapping in a tokamak. As pointed out by $\mathrm{Chang}^{2}{ }^{2}$ the in-out potential asymmetry leads to an electrostatic potential well either on the inside or on the outside of a tokamak, depending on the sign of the potential variation and the charged particle species considered. As the trapped particle effects are crucial to neoclassical transport in the low collisionality regime, we briefly reexamine particle trapping in the presence of a poloidal potential variation of order $\epsilon$ in a multispecies plasma. Following Chang, ${ }^{2}$ we consider particle trapping when (i) $e_{j} \Phi_{\text {in }}<e_{j} \Phi_{\text {out }}$ and (ii) $e_{j} \Phi_{i n}>e_{j} \Phi_{o u t}$, where $\Phi_{\text {in }}$ and $\Phi_{\text {out }}$ are the potential on the high field and low field side of the tokamak respectively, and $e_{j}=Z_{j} e$ is the charge on the species under consideration. Here, $Z_{j}=-1,1, Z$ for the electron, main ion, and impurity ion respectively.

A. $e_{j} \Phi_{\text {in }}<e_{j} \Phi_{\text {out }}$

This situation obtains for ions during ICRH (or NBI) or electrons during ECRH. In the present case, particle trapping can be separated into two categories. The first type, called B-trapping, ${ }^{2}$ is the usual magnetic trapping on the low field side modified by the electrostatic potential. The second type of trapping, called E-field trapping, ${ }^{2}$ occurs on the inside of the torus and is due to the potential well. For a charged particle of mass $m_{j}$, we define the kinetic energy, the total energy, and the magnetic 
moment per unit mass as $w=v^{2} / 2, E=w+e_{j} \Phi / m_{j}$, and $\mu=v_{\perp}^{2} / 2 B$ respectively. Here, $B$ is the total magnetic field, $v_{\perp}$ and $v$ are the perpendicular (to the magnetic field) and total velocity of the particle respectively.

\section{B-trapping}

Using the conservation of energy and adiabaticity of magnetic moment, and following the same steps as in Ref. 2, it can be shown that for B-trapping,

$$
S_{\|}^{2} \leq \epsilon(1+\cos \theta)\left(S_{\perp}^{2}-\left|Z_{j}\right| X_{0}\right)
$$

where $S_{\|}=v_{\|} / v_{t h, j}, S_{\perp}=v_{\perp} / v_{t h, j}$, and $\epsilon X_{0}=\left(e \Phi_{c} / T\right)$. Here, $v_{\|}$is the velocity parallel to the magnetic field and $v_{t h, j}=2 T_{j} / m_{j}$. We have assumed $B=B_{0} / 1+\epsilon \cos \theta$ and $e_{j} \tilde{\Phi}(\theta)=e_{j} \Phi_{c} \cos \theta\left(e_{j} \Phi_{c}>0\right)$. The angle $\theta$ is the poloidal angle measured from the outside of the tokamak. We further note that in the present work $X_{0} \sim O(1)$. At this stage, we assume that $T_{e} \simeq T_{i} \simeq T_{Z}=T$, where the subscripts $e, i, Z$ refer to electron, main ion, and impurity ion respectively. Defining the pitch angle variable $\lambda=\mu B_{0} / E$, we identify the B-trapped region in the $\lambda-E$ space as

$$
(1-\epsilon) \leq \lambda \leq(1+\epsilon)\left[1-\frac{e_{j} \Phi(0)}{m_{j} E}\right]
$$

In deriving Eq. (2), we have set $\Phi(\pi)=0$ and $e_{j} \tilde{\Phi}(0)>0$.

\section{E-trapping}

It can be further shown that for E-trapping,

$$
S_{\|}^{2} \leq \epsilon(1-\cos \theta)\left(\left|Z_{j}\right| X_{0}-S_{\perp}^{2}\right)
$$

The trapped region in the $\lambda-E$ space is given by

$$
(1+\epsilon)\left[1-\frac{e_{j} \Phi(0)}{m_{j} E}\right] \leq \lambda \leq 1-\epsilon
$$

We note that the minimum energy a particle can have is $E_{0}=e_{j} \Phi / m_{j}$. The trapped particle regions for B-trapping and E--trapping in the $S_{\|}-S_{\perp}$ and $\lambda-E$ space are shown in Figs. 1 and 2. The dotted lines show the trapped particle region for $\Phi=0$. 


\section{B. $e_{j} \Phi_{\text {in }}>e_{j} \Phi_{\text {out }}$}

. This scenario holds good for ions during ECRH and electrons during ICRH. When $e_{j} \Phi_{\text {in }}>e_{j} \Phi_{\text {out }}$, only B-trapping is present. ${ }^{2}$ Letting $e_{j} \Phi(\theta)=-e_{j} \Phi_{c} \cos \theta\left(e_{j} \Phi_{c}>0\right)$ and proceeding as before, we determine the boundaries of the trapped particle region in the $S_{\|}-S_{\perp}$ space as

$$
S_{\|}^{2} \leq \epsilon(1+\cos \theta)\left(S_{\perp}^{2}+\left|Z_{j}\right| X_{0}\right)
$$

In the $\lambda-E$ space, setting $e_{j} \Phi(0)=0$, the trapped particle region is bounded by

$$
(1-\epsilon)\left[1-\frac{e_{j} \Phi(\pi)}{m_{j} E}\right] \leq \lambda \leq(1+\epsilon)
$$

Figs. 3 and 4 show the trapped particle region in the velocity $\left(S_{\|}-S_{\perp}\right)$ space and $\lambda-E$ space. (We note that the trapped particle region depicted on the $\lambda-E$ space in Fig. 7 in Ref. 2 differs significantly from that shown on Fig. 4.)

The expressions for the trapped particle region [Eqs. (1)-(6)] differ from the corresponding expressions in Ref. 2 due to the presence of $\left|Z_{j}\right|$ (or $e_{j}$ ). In a simple plasma, $\left|Z_{j}\right|=1$ and Eqs. (1)-(6) reduce to the corresponding expressions in Ref. 2. In the presence of an impurity species with charge $Z$, the effect of a poloidal potential variation is greater on the impurity ions than on the main ions. Indeed, for moderate to large $Z$ impurities, or when the potential variation is large, i.e. $X_{0} \gg 1$, most of the impurity ions are trapped in the potential well. The difference in the trapping fraction of the impurity ions and the main ions alters the collisional coupling between the main ion and the impurity species, causing a significant change in the necclassical transport coefficients in an impure plasma.

\section{SOLUTION OF THE DRIFT KINETIC EQUA- TION IN THE BANANA REGIME}

We begin by considering the first order (in poloidal gyroradius) version of the drift kinetic equation (DKE) $)^{2,6,7.5}$ for the species $j$,

$$
\mathbf{v}_{\|} \cdot \nabla f_{j 1}+\mathbf{v}_{D_{j}} \cdot \nabla f_{j 0}+\frac{e_{j}}{m_{j}} E_{\|} v_{\|} \frac{\partial f_{j 0}}{\partial E}=\sum_{k} C_{j k}\left(f_{j 1}, f_{k 1}\right)
$$




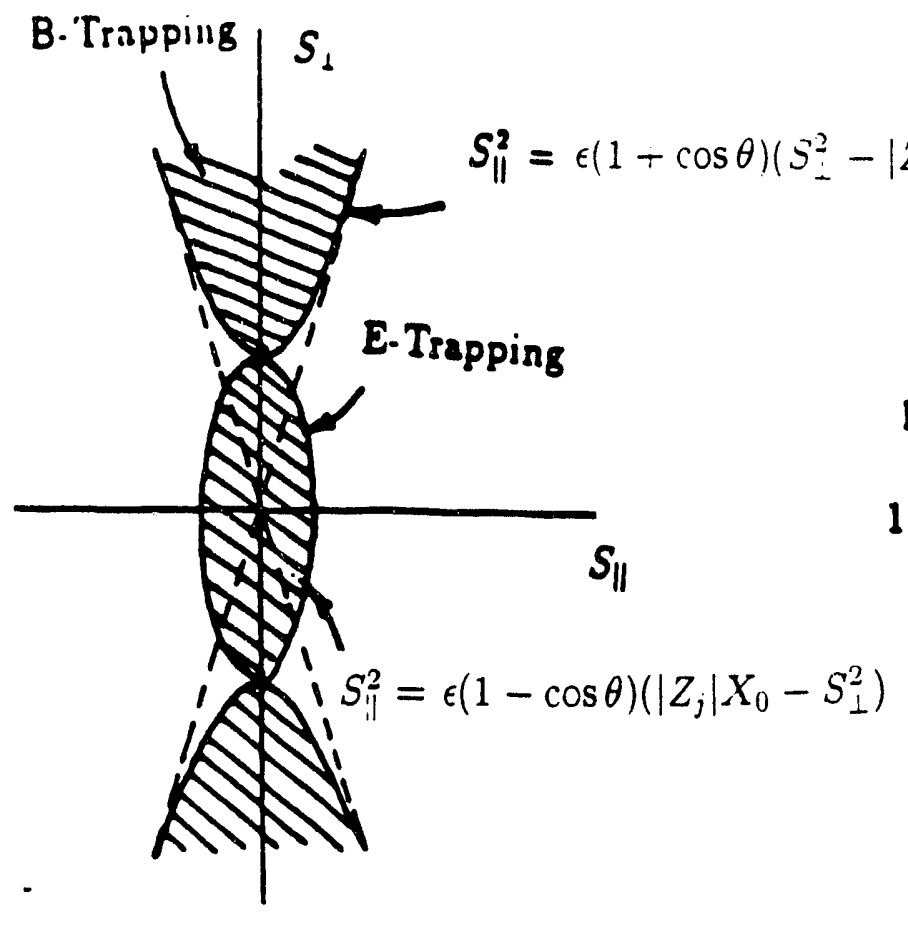

Figure 1

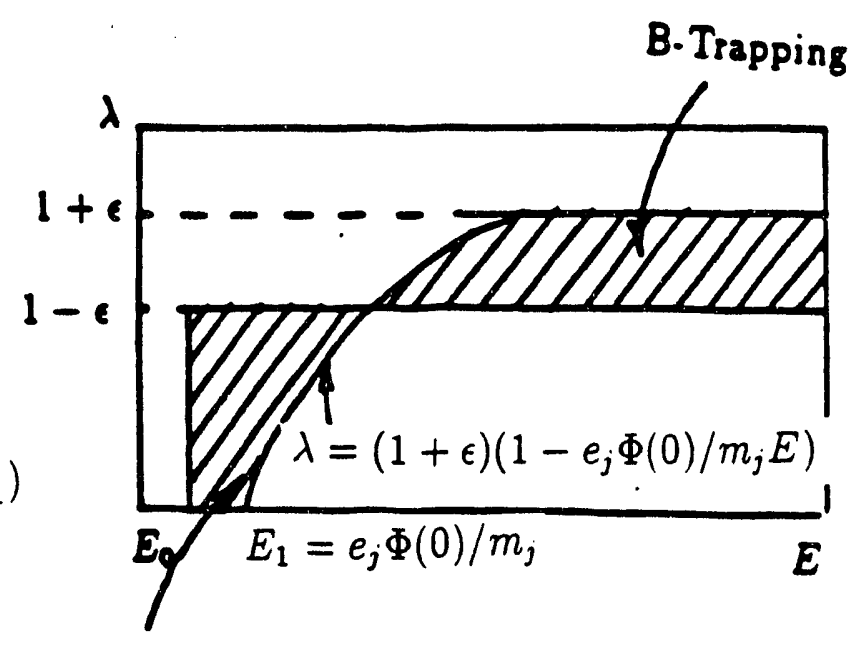

E-Trapping

Figure 2

Particle Trapping when $e_{j} \Phi_{\text {in }}<e_{j} \Phi_{\text {out }}$

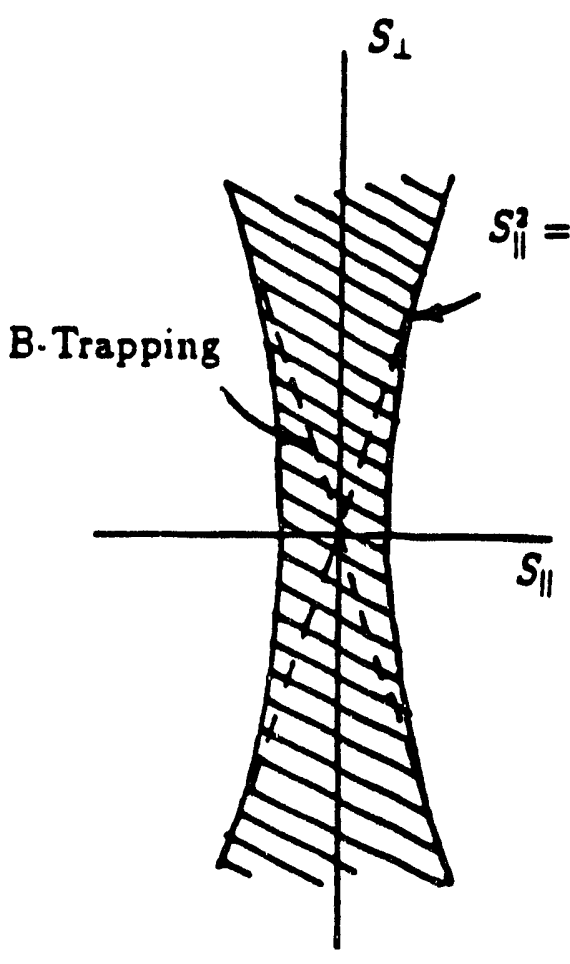

Figure 3

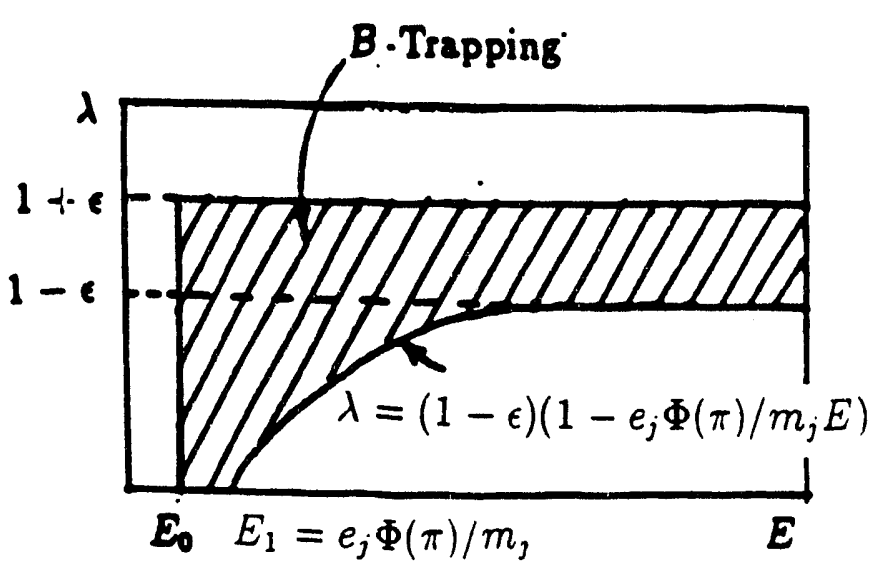

Figure 4

Particle Trapping when $\epsilon_{j} \Phi_{i n}>e_{j} \Phi_{\text {out }}$ 
where the equilibrium distribution function $f_{j 0}$ is given by

$$
f_{j 0}=n_{j 0}\left(\frac{m_{j}}{2 \pi T_{j}}\right)^{3 / 2} \exp \left(-\frac{m_{j} E^{*}}{T_{j}}\right)
$$

Here, due to the presence of a significant poloidal electric field, the energy $E^{*}=$ $w+e_{j} \tilde{\Phi}(\theta) / m_{j}$. Furthermore, $n_{j 0}$ is the flux surface averaged density. For a large aspect ratio tokamak, we define the flux surface operator as

$$
<x>=\frac{1}{2 \pi} \int_{0}^{2 \pi} h x d \theta \simeq \frac{1}{2 \pi} \int_{0}^{2 \pi}(1+\epsilon \cos \theta) x d \theta
$$

For the drift velocity, we use the small $\beta$ result ${ }^{6.2}$

$$
\mathbf{v}_{D j}=-v_{\|} \hat{n} \times \nabla\left(\frac{v_{\|}}{\Omega_{j}}\right)
$$

where $\Omega_{j}=e_{j} B / m_{j}$ and the gradient is taken at constant $E^{*}$ instead of at constant $w$ as in the standard neoclassical theory in the absence of a significant poloidal potential variation. $E_{\|}$is the parallel electric field and $f_{j 1}$ is the first order (in poloidal gyroradius) correction to the distribution function.

We note that in obtaining the drift kinetic equation it is customary to treat quantities which do not explicitly depend upon the gyrophase as constants. ${ }^{8}$ In the presence of a wave heating mechanism, some quantities like $\mathbf{E}_{\perp}$ may not be independent of the gyrophase throughout the plasma volume. We, however, treat these quantities as gyrophase independent for the following reasons: 1) In many wave heating scenarios, particles of only one species participate in the energy absorption process. For instance, during ECRH, only electrons take part in the energy absorption process, while during ICRH, ions are the main participants. (We note that in some instances, electrons can also participate via Landau damping and transit time magnetic pumping.) Furthermore, in many wave heating mechanisms, such as ICRH (minority heating), only a small fraction of particles participate in wave heating. 2) In most wave heating scenarios, the launched wave has $k_{\|} \neq 0$. This means that only a fraction of the particles satisfying the resonance condition $\omega-k_{\|} v_{\|}=0$ participate in wave heating. In addition, the resonance zone where $\mathbf{E}_{\perp}$ may not be zero is confined to a thin region across a vertical plane. Hence, for the bulk of the particles over most of the plasma volume, $\mathbf{E}_{\perp}=0$ is a good assumption. 
With this treatment, we proceed with the solution of DKE. The present treatment differs from that used by Chang ${ }^{2}$ in two ways:

1. We use an analytical procedure of the type used by Hirshman et al. ${ }^{5}$ and Connor, ${ }^{7}$ in contrast with the variational procedure used by Chang. ${ }^{2}$

2. We use the approximate collision operator developed by Hirshman et al. ${ }^{9.5}$ This collision operator has the form

$$
C_{j k}\left(f_{j 1}, f_{k 1}\right)=\nu_{j k}^{D} \mathcal{L} f_{j 1}+\frac{2 v_{\|} r_{k j}}{v_{t h, j}^{2}} \nu_{j k}^{S} f_{j 0}+\left[\nu_{j k}^{D}-\nu_{j k}^{S}\right] \frac{v_{\|} u_{j 1}(v)}{v^{2}} f_{j 0}
$$

Various terms in this equation are defined in Appendix A. The principal difference between this operator and similar operators used by other investiga$\operatorname{tors}^{7,2,10}$ is the distinction between the pitch angle scattering frequency $\left(\nu_{j k}^{D}\right)$ and the slowing down frequency $\left(\nu_{j k}^{S}\right)$. Many of the properties of this operator have been described in Ref. 5. However, for our purposes, the two most important properties of this operator are: (i) its ability to treat collisions between species of arbitrary mass difference and (ii) its capability to treat the plasma in a tokamak with an arbitrary aspect ratio. The second property is particularly important because, in the presence of a significant poloidal electric field, the smallness of the trapped particle region in a large aspect ratio tokamak is destroyed in the low energy region of the velocity space (see Figs. 1-4). Hence, in the low energy region $\left(v<v_{t h, j}\right)$, the trapped particle fraction is close to 1 , while it is still small in the high energy region. The above collision operator is suited for modeling this scenario.

The solution of the DKE is obtained by adopting the procedure used by Hirshman et al. ${ }^{5}$ The aim is to reduce the DKEs for $\mathbf{n}$-species to a set of coupled algebraic equations for the restoring coefficients $<r_{k j}>$ in the banana regime. Here, we define the collisionality parameter as $\nu_{*, j}=\tau_{B} / \tau_{e f f}$, where $\tau_{B}$ is the typical time for a particle to complete the trapped orbit and $\tau_{\text {eff }}$ is the effective time for the trapped particle to scatter out of the banana orbit. Writing

$$
f_{j 1}=-\frac{m_{j}}{e_{j}} \frac{\sigma q}{B_{\theta}} \frac{\partial f_{j 0}}{\partial r}+g_{j}
$$


where $q=\left|v_{\|}\right|$and $\sigma= \pm$ is the sign of the parallel velocity, and expanding $g_{j}$ in terms of the collisionality parameter ${ }^{5} \nu_{* j}<1$, we obtain, to the first order in $\nu_{*, j}$,

$$
\frac{\sigma q}{r} \Theta \frac{\partial g_{j}^{1}}{\partial \theta}=C\left(f_{j 1}\right)+\frac{e_{j}}{T_{j}}(\sigma q) E_{\|} f_{j 0}
$$

Here, $\Theta=B_{\theta} / B$ where $B_{\theta}=B_{\theta}^{0} /(1+\cos \theta)$ is the poloidal component of the magnetic field. We note that the lowest order (in $\nu_{*, j}$ ) solution $g_{j}^{0}$ is again independent of the poloidal angle $\theta$. In the absence of a significant poloidal electric field, the above equation can be integrated in the trapped and untrapped regions to eliminate the unknown function $g_{j}^{1}{ }^{7,5}$ In the present case, due to the presence of a significant poloidal variation in the potential, the collision frequencies and the velocity integrals $u_{j 1}(v)$ and $r_{k j}$ depend upon the poloidal angle. This is due to the poloidal angle dependence of the velocity $v$, which is given by $v^{2}=2\left[E^{*}-e \tilde{\Phi}(\theta) / m\right]$. We can divide the untrapped particle region into (i) untrapped particles forming a part of the boundary layer (marginally untrapped particles) and (ii) well untrapped particles. In the present analysis, we will not carry out a detailed boundary layer analysis. We consider only the untrapped particles with a large kinetic energy in comparison with their potential energy. Hence, we treat the velocity to be independent of poloidal angle for these particles. With this, Eq. (13) can be integrated to obtain ${ }^{7.5}$

$$
\frac{\partial g_{j}^{0}}{\partial \mu}= \begin{cases}-\left(B^{0} /<v_{\|}>\right)\left[\left(m_{j} / e_{j} B_{\theta}^{0}\right) \partial f_{j 0} / \partial r\right. \\ +\left(m_{j} f_{j 0} / T_{j} \nu_{j}^{D}\right) \sum_{k}<r_{k j}>\nu_{j k}^{S}+\left(1-\nu_{j}^{S} / \nu_{j}^{D}\right)\left(<u_{j 1}(v)>/ v^{2}\right) f_{j 0} \\ \left.+\left(e_{j} f_{j 0} / T_{j} \nu_{j}^{D}\right) E^{0}\right] & \text { for passing particles } \\ 0 & \text { for trapped particles }\end{cases}
$$

Here, $\nu_{j}^{S}=\sum_{k} \nu_{j k}^{S}, \nu_{j}^{D}=\sum_{k} \nu_{j k}^{D}$, and $E^{0}=<E_{\|}>$. Upon using the Eqs. (14) and (12) in the expressions for $u_{j 1}(v)$ and the restoring coefficients $r_{k j}$, we obtain ${ }^{11,5}$

$$
\begin{aligned}
<r_{k j}>= & -\frac{1}{\left\{\nu_{k j}^{S}\right\}}\left(\frac{T_{k}}{e_{k} B_{\theta}^{0}}\right)\left[\left\{\nu_{k j}^{S}\right\}\left(A_{1 k}+\frac{e_{k} \Phi^{\prime}}{T_{k}}\right)+\left\{\nu_{k j}^{S}\left(\frac{m_{k} E^{*}}{T_{k}}\right)\right\} A_{2 k}\right] \\
& +\frac{1}{\left\{\nu_{k j}^{S}\right\}}\left(\frac{T_{k}}{e_{k} B_{\theta}^{0}}\right)\left[\left\{\frac{\nu_{k j}^{S} \nu_{k}^{S}}{\nu_{k \Phi}} f_{c \Phi}\right\}\left(A_{1 k}+\frac{e_{k} \Phi^{\prime}}{T_{k}}\right)\right. \\
& \left.+\left\{\frac{\nu_{k j}^{S} \nu_{k}^{S}}{\nu_{k \Phi}}\left(\frac{m_{k} E^{*}}{T_{k}}\right) f_{c \Phi}\right\} A_{2 k}\right] \\
& +\frac{e_{k}}{m_{k}} A_{3} \frac{1}{\left\{\nu_{k j}^{S}\right\}}\left\{\frac{\nu_{k j}^{S}}{\nu_{k \Phi}} f_{c \Phi}\right\}+\sum_{i} \frac{1}{\left\{\nu_{k j}^{S}\right\}}\left\{\frac{\nu_{k j}^{S} \nu_{k l}^{S}}{\nu_{k \Phi}} f_{c \Phi}\right\}\left\langle r_{l k}\right\rangle
\end{aligned}
$$


Using the same notation as Hirshman et al., ${ }^{5}$ we have defined the "driving forces" as

$$
A_{1 k}=\frac{n_{k 0}^{\prime}}{n_{k 0}}-\frac{3}{2} \frac{T_{k}^{\prime}}{T_{k}} ; \quad A_{2 k}=\frac{T_{k}^{\prime}}{T_{k}} ; \quad A_{3}=E^{0}
$$

where the prime denotes derivative with respect to $r$.

In Eq. (15), the effect of the poloidal electric field is contained in $f_{c \Phi}\left(=1-f_{t \Phi}\right)$, $\nu_{k \Phi}=f_{c \Phi} \nu_{k}^{S}+f_{t \Phi} \nu_{k}^{D}$, and $E^{*}\left(=v^{2} / 2+e \Phi(\theta) / m\right)$. The most significant effect is contained in the neoclassical factor $f_{t \Phi}\left(=1-f_{c \Phi}\right)$. The quantity $f_{t \Phi}$ is analogous to the quantity $f_{t}$ defined in Ref. 5. Indeed, for $\tilde{\Phi}(\theta) \simeq 0, f_{t \Phi}=f_{t}$. The factor $f_{t \Phi}$ contains the effect of modification of the boundary between the trapped and untrapped regions in the velocity space due to the poloidal electric field. For a large aspect ratio tokamak, we obtain the following approximate expressions for $f_{t \Phi}$ (see Appendix B for details).

For $e_{j} \Phi_{\text {in }}<e_{j} \Phi_{\text {out }}$,

$$
f_{t \Phi} \simeq f_{t}\left|1-\frac{\left|Z_{j}\right| X_{0}}{x_{j}^{2}}\right|^{1 / 2}
$$

and, for $e_{j} \Phi_{i n}>e_{j} \Phi_{\text {out }}$,

$$
f_{t \Phi} \simeq f_{t}\left|1+\frac{\left|Z_{j}\right| X_{0}}{x_{j}^{2}}\right|^{1 / 2}
$$

where $x_{j}^{2}=m_{j} w / T$. In the absence of a poloidal electric field, $f_{t \Phi}=f_{t}{ }^{5}$

When $\tilde{\Phi}(\theta) \simeq 0$, the Eq. (15) reduces to Eq. (26) in Ref. 5. Noting that $\nu_{k \Phi}=f_{c \Phi} \nu_{k}^{S}+f_{t \Phi} \nu_{k}^{D}$,

$$
\frac{f_{c \Phi} \nu_{k}^{S}}{\nu_{k \Phi}}=1-\frac{f_{t \Phi} \nu_{k}^{D}}{\nu_{k \Phi}}
$$

Using this result and summing the Eq. (15) over $\mathbf{j}$, we obtain, after changing the dummy index $l$ to $j$, the following useful expression ${ }^{7}$

$$
\begin{aligned}
\sum_{j}\left\{\nu_{k j}^{S}\right\}\left[<r_{k j}>-<r_{j k}>\right]= & \frac{e_{k} E^{0}}{m_{k}}-\frac{T_{k}}{e_{k} B_{\theta}^{0}}\left[\left\{\frac{\nu_{k}^{S} \nu_{k}^{D}}{\nu_{k \Phi}} f_{t \Phi}\right\}\left(A_{1 k}+\frac{e_{k} \Phi^{\prime}}{T_{k}}\right)\right. \\
& \left.+\left\{\frac{\nu_{k}^{S} \nu_{k}^{D}}{\nu_{k \Phi}}\left(\frac{m_{k} E^{*}}{T_{k}}\right) f_{t \Phi}\right\} A_{2 k}\right]-\frac{e_{k}}{m_{k}} A_{3}\left\{\frac{\nu_{k}^{D}}{\nu_{k \Phi}} f_{t \Phi}\right\} \\
& -\sum_{j}\left\{\frac{\nu_{k j}^{S} \nu_{k}^{D}}{\nu_{k \Phi}} f_{t \Phi}\right\}<r_{j k}>
\end{aligned}
$$

This equation is useful in determining the particle fluxes. 


\section{PARTICLE TRANSPORT}

The radial particle flux is given by

$$
\Gamma_{j} \equiv\left\langle\int d^{3} v f_{j} v_{d r, j}\right\rangle
$$

where $v_{d r, j}$ is the radial component of the drift for the species $j$. Using the expression for the drift [Eq. (10)] and the previously obtained solution for $f_{j 1}$, the particle flux can be expressed in terms of the restoring coefficients ${ }^{5}$ as

$$
\Gamma_{j \Phi}=\sum_{k} \frac{m_{j} n_{j 0}\left\{\nu_{j k}^{S}\right\}}{e_{j} B_{\theta}^{(0}}\left[<r_{j k}>-<r_{k j}>\right]-\frac{n_{j 0} E^{0}}{B_{\theta}^{(0}}
$$

Here, the subscript $\Phi$ denotes the presence of a significant poloidal electric field. Using Eq. (21) and the momentum conservation valation ${ }^{5} m_{j} n_{j}\left\{\nu_{j k}^{S}\right\}=m_{k} n_{k}\left\{\nu_{k j}^{S}\right\}$, it can be easily shown that the particle fluxes a bipolar, i.e. $\sum_{j} e_{j} \Gamma_{j \Phi}=0$. Using the expression (19) in Eq. (21), the particle flux is written as

$$
\begin{aligned}
\Gamma_{j \Phi}= & -\frac{m_{j} n_{j 0} T_{j}}{e_{j}^{2} B_{\theta}^{0^{2}}}\left[\left(A_{1 j}+\frac{e_{j} \Phi^{\prime}}{T_{j}}\right)\left\{f_{t \Phi} \frac{\nu_{j}^{S} \nu_{j}^{D}}{\nu_{j \Phi}}\right\}+A_{2 j}\left\{f_{t \Phi} \frac{\nu_{j}^{D} \nu_{j}^{S}}{\nu_{j \Phi}}\left(\frac{m_{j} E^{*}}{T_{j}}\right)\right\}\right] \\
& -\frac{m_{j} n_{j 0}}{e_{j} B_{\theta}^{0}} \sum_{k}\left\{\frac{f_{t \Phi} \nu_{j}^{D} \nu_{j k}^{S}}{\nu_{j \Phi}}\right\}<r_{k j}>-\frac{n_{j 0} A_{3}}{B_{\theta}^{0}}\left\{\frac{f_{t \Phi} \nu_{j}^{D}}{\nu_{j \Phi}}\right\}
\end{aligned}
$$

where the restoring coefficients are given by Eq. (15). We note here that the procedure used for obtaining the above expression for the particle flux in the presence of a poloidal electric field is similar to that used by Connor ${ }^{7}$ and Hirshman et al. ${ }^{5}$ The above expression for the particle flux differs from the corresponding expression [Eq. $(30 \mathrm{a})]$ in Ref. 5 in that $f_{t \Phi}$ is energy dependent and $E^{*}=v^{2} / 2+e \tilde{\Phi}(\theta) / m$. When $\tilde{\Phi}(\theta)=0$, the above expression reduces to Eq. (30a) in Ref. 5 .

As the restoring coefficients are linear in the driving forces $A_{1 k}, A_{2 k}, A_{3}$, and $\Phi^{\prime}$, the particle flux due to each of the driving forces may be obtained separately. ${ }^{5}$ The radial electric field does not contribute to the particle fluxes. Hence, we consider the particle flux due to $A_{1 k}, A_{2 k}$, and $A_{3 k}$.

\section{A. Response to the gradient terms}

We first consider the diffusive fluxes in response to the gradient terms $A_{1 k}$ and $A_{2 k}$. The expression for the parti le flux [Eq. (22)] indicates that, to determine the particle 
flux to the order $f_{t \varphi}$, it is sufficient to determine the restoring coefficients $\left\langle r_{k j}>\right.$ to the lowest order in $f_{t \phi}$. This fact and $t^{2}$ form of the equation for the restoring coefficients, Eq. (15), suggest the following expansion for $\left\langle r_{j k}\right\rangle$

$$
<r_{k j}>=V_{\Phi}+f_{i \Phi}<r_{k j}>_{1}+\cdots
$$

where, to the zeroth order in $f_{t \Phi}$, the restoring coefficients of all the species equal a common toroidal rotation velocity $V_{\Phi}$. Such an expansion has been used by Hirshman et al. ${ }^{5}$ to solve for the particle fluxes. As discussed in Ref. 5 , the validity of such an expansion depends upon two conditions.

1. Collisional coupling among passing particles of the various species should be sufficiently strong to establish a common flow. This condition is usually met in a device even with a small impurity concentration.

2. The trapped particle fraction is sufficiently small. The quantity $f_{1}$ is proportional to the fraction of trapped particles. In a large aspect ratio tokamak ( $\epsilon \ll$ 1), when $\tilde{\Phi}(\theta) \simeq 0$, this condition is satisfied. In this case, $f_{t \phi}=f_{t} \sim O(\sqrt{\epsilon})$. In the presence of a poloidal electric field, we may obtain one of the two scenarios outlined below.

(a) When $X_{0} \simeq 1$, for electrons, ions, and lighter impurities the following discussion is valid.

In the presence of a significant poloidal electric field, we have found that the boundary between the trapped-untrapped particle regions is a function of the particle energy (see Section II.). Referring to Figs. 1-4, we find that, for the low energy particles, the extent of the trapped particle region in the $\lambda$ direction is not small. Analytically, this difficulty manifests itself in the velocity dependence of the quantity $f_{t \boldsymbol{t}}$, which is velocity independent when $\bar{\Phi}(\theta)=0$. As a result, velocity integrals of the type $\left\{f_{t} F(v)\right\} \neq f_{t}\{F(v)\}$ where $F(v)$ is a: arbitrary function of velocity. Actually $\left\{f_{t} F(v)\right\}>f_{t}\{F(v)\}$, especially for large values of the poloidal electric field (i.e. $X_{0}>1$ ). However, we note from Figs. 1-4 that the extent of the region with increased trapping is small in the $E$ direction 
for a large Espect ratio tokamak. Furthermore, the contribution of the low velocity region with enhanced trapping to the velocity integrals of the type $\left\{f_{t \Phi} F(c ;\}\right.$ is small (see Appendix $\mathrm{C}$ ). We, therefore, assume that $\left\{f_{t \Phi} F(v)\right\} \sim O\left(f_{t}\right)$. Specifically, we assume that the enhancement of velocity integrals of the type $\left\{f_{t \Phi} F(v)\right\}$ over similar integrals when $\tilde{\Phi}(\theta)=0$ is only by a factor less than an order of magnitude.

(b) When $X_{0} \simeq 1$, for a heavy impurity $\left(Z_{j}=Z \gg 1\right)$, the quantity $\left|1 \pm \frac{Z X_{0}}{x_{z}^{2}}\right|^{1 / 2}$ can be quite large and $f_{t \Phi}$ can approach unity for moderate values of $\sqrt{\epsilon}$. Physically, this means that most of the impurity particles are trapped in the electrostatic potential well. If this situation obtains, the expansic Eq. (23) is not valid.

From now on, we concentrate on those situations where $f_{t \Phi}<1$. With this, we use the expansion (23) in the expression for particle fluxes. Using only the lowest order (in $f_{t \Phi}$ ) restoring coefficient $V_{\Phi}$ in $\mathrm{E}_{2}$. (22) and the ambipolarity of the particle fluxes, we obtain the common toroidal rotation velocity as

$$
V_{\Phi}=-\frac{\sum_{j}\left(m_{j} n_{j} T_{j} / e_{j} B_{\theta}^{0}\right)\left[A_{1 j}\left\{f_{t \Phi} \nu_{j}^{S} \nu_{j}^{D} / \nu_{j \Phi}\right\}+A_{2 j}\left\{f_{t \Phi} \nu_{j}^{S} \nu_{j}^{D} / \nu_{j \Phi}\left(m_{j} E^{*} / T_{j}\right)\right\}\right]}{\sum_{j} m_{j} n_{j}\left\{f_{t \Phi} \nu_{j}^{D} \nu_{j}^{j} / \nu_{j \Phi}\right\}}
$$

Using this expression in Eq. (22), we obtain the following expression for the diffusive particle fluxes.

$$
\Gamma_{j \Phi}\left(A_{1}, A_{2}\right)=\sum_{k ; n=1,2} L_{1 n, \Phi}^{j k} A_{n k}
$$

where

$$
\begin{aligned}
L_{1, \Phi}^{j j} & =-\left(\frac{\sum_{l \neq j} m_{l} n_{l}\left\{f_{t \Phi} \nu_{l}^{D} \nu_{l}^{S} / \nu_{l \Phi}\right\}}{\sum_{l} m_{l} n_{l}\left\{f_{t \Phi} \nu_{l}^{D} \nu_{l}^{S} / \nu_{l \Phi}\right\}}\right) L_{j \Phi} \\
L_{12, \Phi}^{j j} & =\frac{\left\{f_{l \Phi} \nu_{j}^{D} \nu_{j}^{S} / \nu_{j \Phi}\left(m_{j} E^{*} / T_{j}\right)\right\}}{\left\{f_{t \Phi} \nu_{j}^{D} \nu_{j}^{S} / \nu_{j \Phi}\right\}} L_{11, \Phi}^{j j} \\
L_{11, \Phi}^{j k} & =\frac{Z_{j}}{Z_{k}} \frac{T_{k}}{T_{j}}\left(\frac{m_{k} n_{k}\left\{f_{t \Phi} \nu_{k}^{D} \nu_{k}^{S} / \nu_{k \Phi}\right\}}{\sum_{l} m_{l} n_{l}\left\{f_{t \Phi} \nu_{l}^{D} \nu_{l}^{S} / \nu_{l \Phi}\right\}}\right) L_{j \Phi} \\
L_{12, \Phi}^{j k} & =\frac{\left\{f_{l \Phi} \nu_{k}^{D} \nu_{k}^{S} / \nu_{k \Phi}\left(m_{k} E^{*} / T_{k}\right)\right\}}{\left\{f_{t \Phi} \nu_{k}^{D} \nu_{k}^{S} / \nu_{k \Phi}\right\}} L_{11, \Phi}^{j k}
\end{aligned}
$$


and

$$
L_{j \Phi}=\frac{m_{j} n_{j} T_{j}}{e_{j}^{2} B_{\theta}^{0^{2}}}\left\{f_{t \Phi} \frac{\nu_{j}^{S} \nu_{j}^{D}}{\nu_{j \Phi}}\right\}
$$

From the above equations, it is easy to see that

$$
\frac{\Gamma_{i}}{\Gamma_{e}} \sim O\left(\sqrt{\frac{m_{i}}{m_{e}}}\right)
$$

Hence, to watisfy anbipolarity, $\Gamma_{Z} \simeq-\left(e_{i}\right) /\left(e_{z}\right) \Gamma_{i}$. Thus, ion and impurity diffusion occur at a comparable rate at the beginning of the discharge. Over a longer time scale, the ion and impurity density and temperature profiles adjust to make the ion and impurity fluxes comparable to the electron flux. When this condition (known as the "stationary state" ) ottains, ambipolarity gives

$$
\Gamma_{e \Phi}=Z_{i} \Gamma_{i \Phi}+Z \Gamma_{Z \Phi}
$$

\section{B. Response to the parallel electric fleld}

Now, we calculate the electron flux due to the parallel electric field. The solution method used here is similar to that used by Connor. ${ }^{7}$ Using Eq. (15), in the absence of $A_{1 k}, A_{2 k}$, and $\Phi^{\prime}$, it can be easily shown that ${ }^{7}$

$$
<r_{e j}>\gg<r_{i j}>,<r_{I j}>
$$

Here, we again have used $\left\{f_{t \Phi} F(v)\right\} \sim O\left(f_{t}\right)$. Using Eq. (15) to obtain $\left\langle r_{e e}\right\rangle$, and using Eq. (22), the radial electron flux due to the parallel electric field can be written as

$$
\Gamma_{e \Phi}\left(A_{3}\right)=-\frac{n_{e 0} A_{3}}{B_{\theta}^{0}}\left[\left\{\frac{f_{t \Phi} \nu_{e}^{D}}{\nu_{e \Phi}}\right\}+\frac{\left\{f_{t \Phi} \nu_{e}^{D} \nu_{e e}^{S} / \nu_{e \Phi}\right\}\left\{\nu_{e e}^{S} / \nu_{e \Phi}\right\}}{\left\{\nu_{e e}^{S}\left(\nu_{e \Phi}-\nu_{e e}^{S}\right) / \nu_{e \Phi}\right\}}\right]
$$

Again, as with the diffusive fluxes, the effect of the poloidal electric field is contained in the factor $f_{t \in}$ and $\nu_{e \Phi}$. When $\tilde{\Phi}(\theta)=0$, our results for the Ware pinch are identical to those obtained by Hirshman et al. ${ }^{5}$ Also, if we set $\nu_{j k}^{S}=\nu_{j k}^{D}$ and $\nu_{e \Phi}=\nu_{e}^{D}$ in Eq. (28), we obtain results identical to those obtained by Connor. ${ }^{7}$

Before we present the results on transport coefficients, we wish to make a few simplifications which greatly facilitate the calculations. 
1. We note that $\nu_{j \Phi}=\left[\nu_{j}^{S}+f_{t \Phi}\left(\nu_{j}^{D}-\nu_{j}^{S}\right)\right]$. In the absence of a poloidal electric field, $\nu_{j}=\left[\nu_{j}^{S}+f_{t}\left(\nu_{j}^{D}-\nu_{j}^{S}\right)\right]$, and, for a large aspect ratio tokamak $\left(f_{t} \ll 1\right)$, we can set $\nu_{j} \simeq \nu_{j}^{S}$ to the lowest order in $f_{t} \cdot{ }^{5}$ When a poloidal electric field is present, we have $f_{t \Phi}=f_{t}|1 \pm| Z_{j}\left|X_{0} / x_{j}^{2}\right|^{1 / 2}$. We observe that in the low energy region where $x=v / v_{t h} \ll 1, f_{t \Phi}$ can be quite large, approaching 1 . It can be shown (see Appendix $\mathrm{C}$ ) that in the large aspect ratio limit, the contribution of this region to velocity integrals of the form $\left\{f_{t \Phi} F(v)\right\}$, where $F(v)$ is a function of collision frequencies, is small. Hence, we set $\nu_{j \Phi} \simeq \nu_{j}^{S}$ while evaluating the velocity integrals.

2. We note that some of the velocity integrals in the above expressions involve $E^{*}=w+e \tilde{\Phi}(\theta) / m$. As the second term in this equation is comparable to the first term only in the low velocity region, and as the contribution of the low velocity region to the velocity integrals of the form $\{F(v)\}$ is small (see Appendix $\mathrm{C}$ ), we use $E^{*} \simeq w$ in evaluating the velocity integrals.

The velocity integrals appearing in the transport coefficients [see Eqs. (25) and (28)] are analytically intractable. Hence, consistent with the approach used by Chang, ${ }^{2}$ we present numerical results for the transport coefficients. We consider a three species plasma in which deuterium is the main ion and the impurity species is carbon. We also set $T_{e} \simeq T_{i} \simeq T_{z}$.

\section{Electron Transport}

\section{Diffusive Flux}

To facilitate comparison of our results with previous results, we write, by rearranging the terms in Eq. (25), the diffusive electrnn flux as

$$
\begin{aligned}
\Gamma_{e \Phi}= & L_{11, \Phi}^{e e}\left(1+\frac{T_{i}}{T_{e} Z_{i}}\right) \frac{n_{e}^{\prime}}{n_{e}}+\left[L_{11, \Phi}^{e e}+L_{11, \Phi}^{e e}\left(y_{e \Phi}-\frac{5}{2}\right)\right] \frac{T_{e}^{\prime}}{T_{e}} \\
& +L_{11, \Phi}^{e e}\left(\frac{T_{i}}{Z_{i} T_{e}}\right)\left(y_{i \Phi}-\frac{3}{2}\right) \frac{T_{i}^{\prime}}{T_{i}}+L_{11, \Phi}^{e e}\left(\frac{T_{i}}{Z_{i} T_{e}}\right)\left(\frac{n_{i}^{\prime}}{n_{i}}-\frac{n_{e}^{\prime}}{n_{e}}\right) \\
& -\frac{Z}{T_{Z}} L_{11, \Phi}^{e Z}\left[\frac{T_{i}}{Z_{i}}\left[\frac{n_{i}^{\prime}}{n_{i}}+\left(y_{i \Phi}-\frac{3}{2}\right) \frac{T_{i}^{\prime}}{T_{i}}\right]-\frac{T_{Z}}{Z}\left[\frac{n_{Z}^{\prime}}{n_{Z}}+\left(y_{Z \Phi}-\frac{3}{2}\right) \frac{T_{Z}^{\prime}}{T_{Z}}\right]\right]
\end{aligned}
$$


Here, we have defined the quantities $y_{j \Phi}$ as

$$
y_{j \Phi}=\frac{\left\{f_{t \Phi} \nu_{j}^{D}\left(m_{j} E^{*} / T_{j}\right)\right\}}{\left\{f_{t \Phi} \nu_{j}^{D}\right\}}
$$

where we have used $\nu_{j \Phi} \simeq \nu_{j}^{S}$. We note that in the absence of impurities, the last two terms in Eq. (29) vanish, and the resulting expression has the same form as the expression for the electron flux obtained by Chang. ${ }^{2}$ In the presence of an impurity species, after the stationary state ${ }^{7}$ has been reached, the last term in Eq. (29) vanishes and it reduces to the form obtained by Connor. ${ }^{7}$ Here, we present the results on electron transport coefficients only after tho stationary state has been reached. Consistent with the approach used by Chang, ${ }^{2}$ we now define the transport enhancement factors as

$$
F_{1 n}^{j k}=\frac{L_{1 n . \Phi}^{j k}}{L_{1 n}^{j k}}
$$

where $n=1,2$. Here, $L_{1 n, \Phi}^{j k}$ and $L_{1 n}^{j k}$ are the transport coefficients with and without a poloidal electric field respectively. $L_{1 n}^{j k}$ is obtained by using $f_{t \Phi}=f_{t}$ in Eqs. (25).

Variation of electron transport enhancement factors $F_{11}^{e e}$ and the quantities $y_{j \Phi}$ $(j=e, i)$ as a function of the poloidal potential variation $X_{0}$ is shown in Figs. 5,6, and 7. We note that the topology of the curves seems to be related to the nature of the factor $f_{t \Phi}$, which is proportional to the trapped particle fraction. As discussed in Section II., when $e_{j} \Phi_{i n}>e_{j} \Phi_{\text {out }}$, B-trapping is enhanced by the potential variation. Behavior of the enhancement factors $F_{11}^{e k}$ seems to reflect this. For $e_{j} \Phi_{i n}<e_{j} \Phi_{\text {out }}$, the situation is somewhat complicated. For the low energy particles, there is a reduction in B-trapping, which, however, is somewhat compensated by the presence of E-trapped particles. It appears that for small values of $X_{0}$, there can be a decrease in the trapped particle fraction which is reflected in the behavior of enhancement factors for small $X_{0}$. It also appears that for large values of $X_{0}$, there can be an increase in the trapped particle fraction. The quantity $y_{j \Phi}$ is related to plasma rotation. It appears that enhanced (or reduced) trapping due to a poloidal electric field causes a decrease (or increase) in the collisional coupling between the species, resulting in a decrease (or increase) in the quantity $y_{j \Phi}$.

We now compare our results, in the absence of impurities, with those obtained 


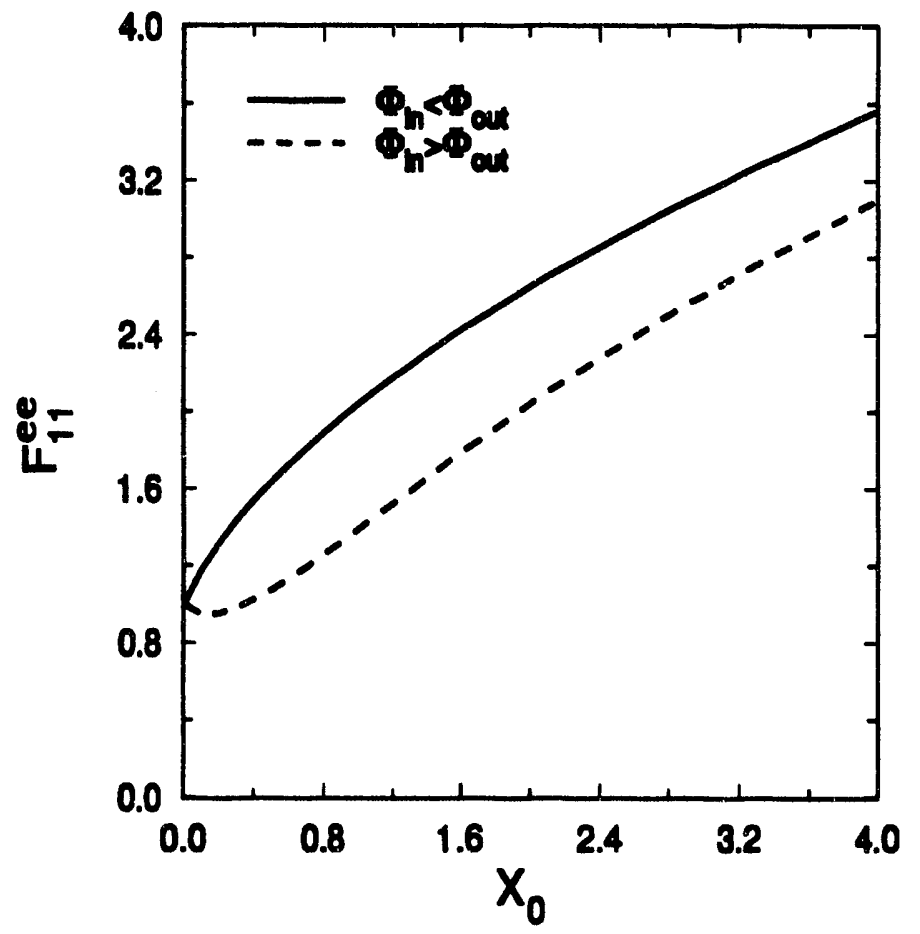

Figure 5: Electron diffusion enhancement factor $F_{11}^{e e}\left(=L_{11 . \Phi}^{e e} / L_{11}^{e e}\right)$ as a function of the magnitude of the poloidal potential variation, $X_{0}$ (Impurity: Carbon; $Z_{\text {eff }}=3.0$ ) 


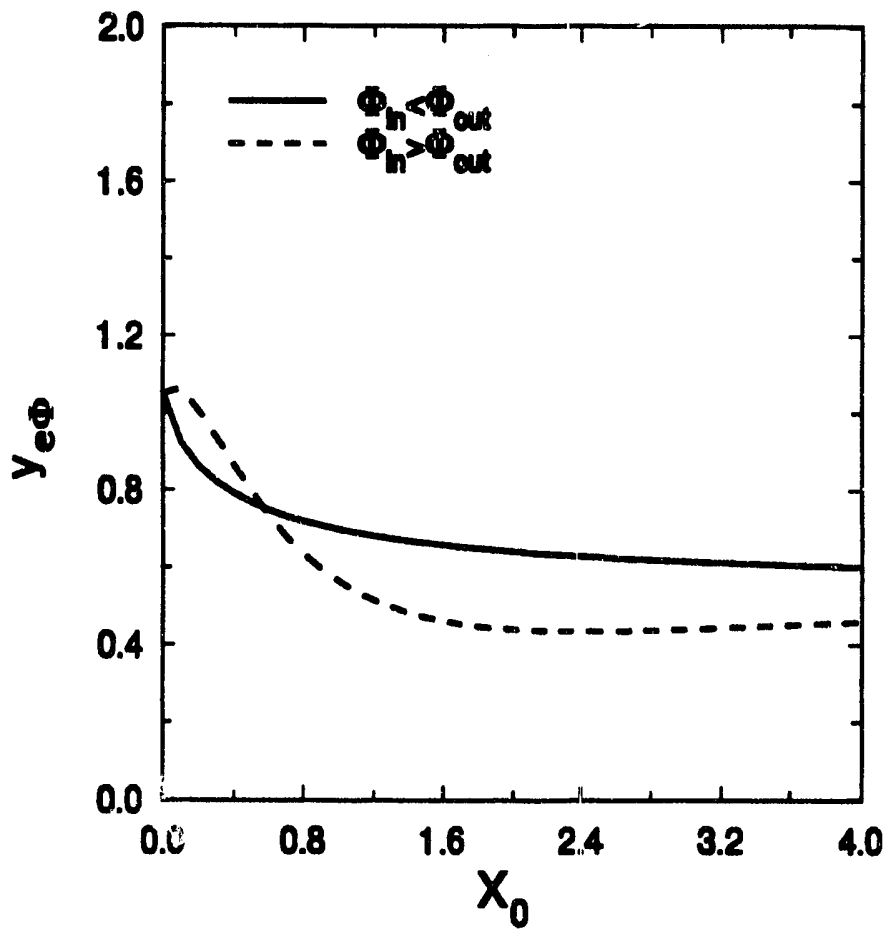

Figure 6: The transport parameter $\eta_{e \Phi}$ as a function of $X_{0}$ (Impurity: Carbon; $Z_{\text {eff }}=3.0$ ) 


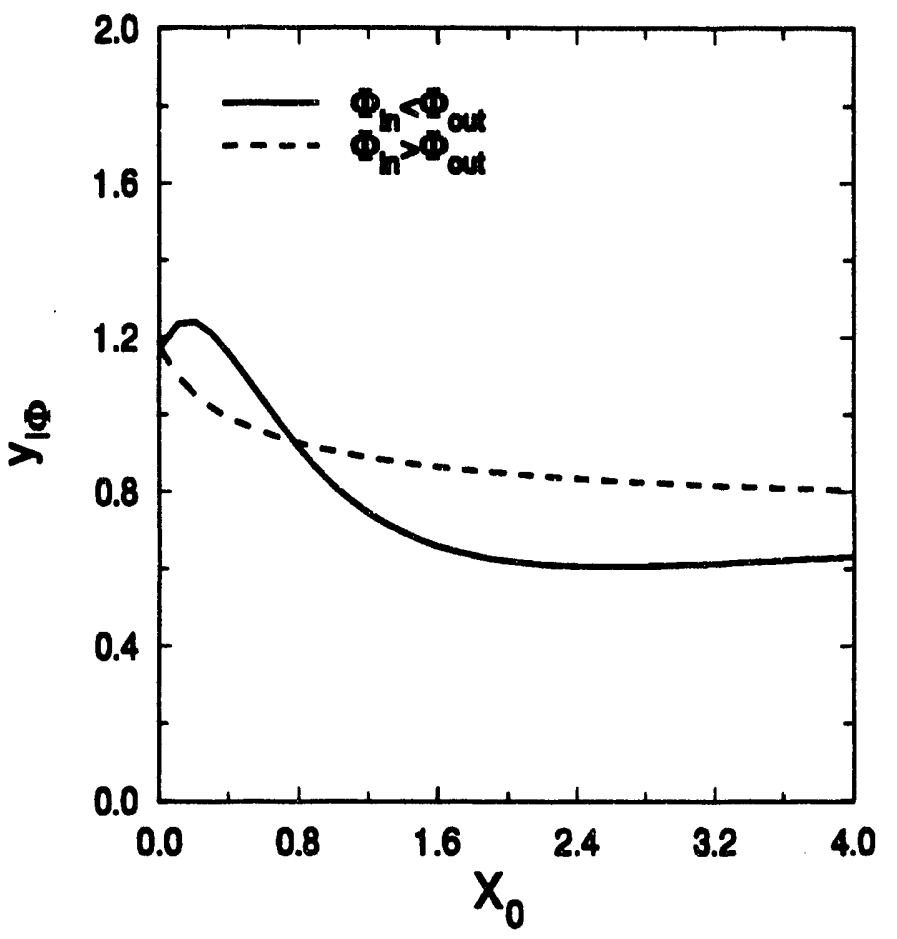

Figure 7: The transport parameter $y_{i \Phi}$ as a function of $X_{0}$ (Impurity: Carbon; $Z_{\text {eff }}=3.0$ ) 
by Chang. ${ }^{2}$ We note that the factor $F_{11}^{e e}$ defined here is equivalent to the factor $F_{11}^{(e)}$ defined in Ref. 2. Also, the quantity $y_{i \Phi}$ defined here is equivalent to the varis ional parameter $y$ defined by Chang. In the absence of impurities, the agreement between $y_{i \Phi}$ and $y$ is very good. However, the result for $F_{11}^{e e}$ obtained in this work differs from that obtained by Chang for $F_{11}^{(e)}$ by $15 \%$ for $X_{0}=4$. The discrepancy is smaller for smaller values of $X_{0}$. (Our calculation of the enhancement factor $F_{11}^{(e)}$,defined by Chang, ${ }^{2}$ using the expressions in Ref. 2 yields a much closer agreement between $F_{11}^{e e}$ and $F_{11}^{(e)}$, the difference being less than $\sim 2 \%$ at $X_{0}=4$.) We note in passing that the transport coefficient $\left(\alpha_{1}, g_{2 e}\right)$ defined in Ref. 2 is equivalent to $L_{11 . \Phi}^{e e}\left(y_{e \Phi}-5 / 2\right)$ in this work, and the comparison between the corresponding enhancement factors yields a result similar to that between $F_{11}^{e e}$ and $F_{11}^{(e)}$.

The presence of an impurity has negligible effect on the transport enhancement factors $F_{11}^{e e}$ and the quantities $y_{j \Phi}$. This seems to be in agreement with the conclusion drawn by Chang. ${ }^{2}$ We note, however, that the presence of an impurity results in two additional terms in the expression for the electron flux [the 4th and 5th terms in Eq. (29)]. Finally, we note that, in the absence of a poloidal electric field, our results on $L_{11}^{e e}$ and $y_{e}$ agree with those obtained by Connor. ${ }^{7}$ However, due to the large impurity mass assumption used in Ref. 7, Connor's result for $y_{i}$ differ from those obtained in this work by $\sim 30 \%$ in the presence of carbon impurity when $\alpha=n_{Z} Z^{2} / n_{i} Z_{i}^{2}=1$.

\section{Ware pinch effect}

We define the Ware pinch enhancement factor as

$$
F_{13}^{e}=\frac{L_{13, \Phi}^{e}}{L_{13}^{e}}
$$

Figure 8 shows the variation of the Ware pinch enhancement factor as a function of $X_{0}$. The Ware pinch enhancement factor is topologically similar to the diffusion enhancement factors. The enhancement, however, is much weaker than that of the diffusion coefficients.

The Ware pinch enhancement factor generally decreases with increasing $Z_{\text {eff }}$. However, when $\Phi_{i n}>\Phi_{\text {out }}$ and for $X_{0} \leq 1$, it remains constant or might even increase slightly. Referring to Eq. (28), the effect of electron-electron collisions is contained 


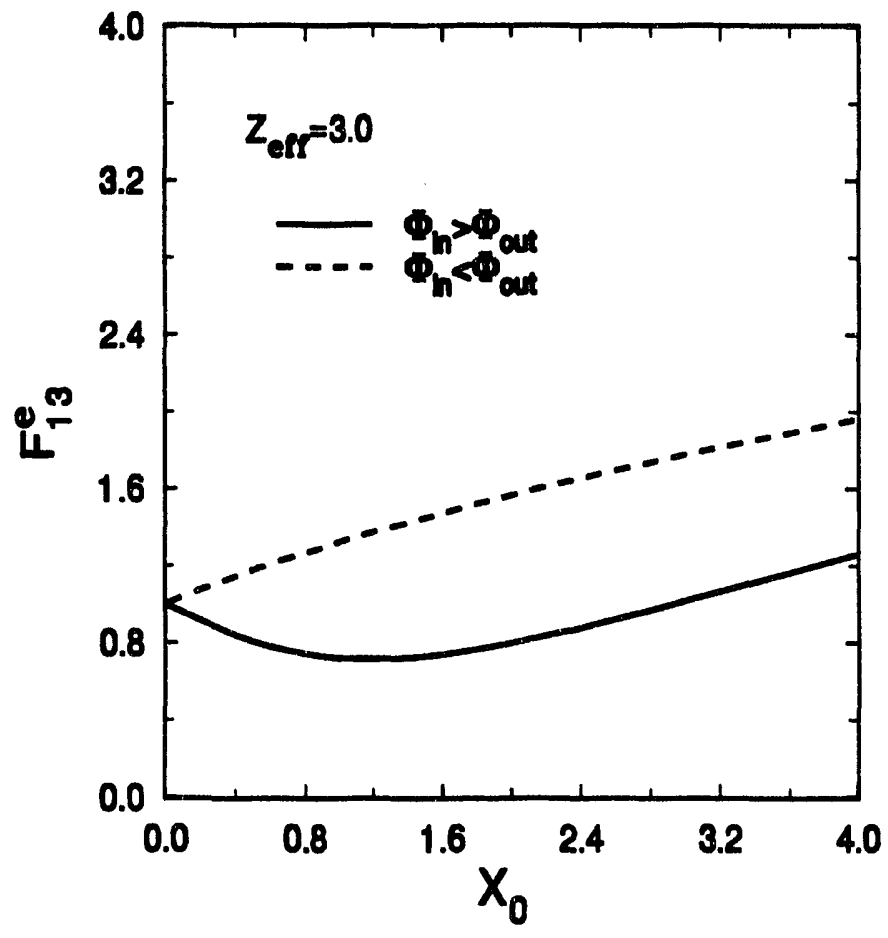

Figure 8: Ware pinch enhancement factor $F_{13}^{e}\left(=L_{13, \Phi}^{e} / L_{13}^{e}\right)$ as a function of $X_{0}$ 
primarily in the second term. It appears that the presence of impurities generally leads to a reduction in the contribution of the electron-electron collisions relative to the first term, leading to a decrease in the Ware Pinch enhancement factor. The behavior of the Ware pinch enhancement factor with increasing $Z_{\text {eff }}$ is similar to the behavior of the neoclassical conductivity reduction factor. We discuss this in Section V.

We have compared our result for the Ware pinch enhancement factor $\left(F_{13}^{e}\right)$ with the result $\left[F_{13}^{(e)}\right]$ obtained by Chang ${ }^{2}$ in the absence of impurities. As with the electron diffusion coefficients, we find that our results differ from those obtained by Chang by $\sim 20 \%$ for $X_{0}=4$ and the enhancement factors calculated here are smaller than those in Ref. 2.

\section{Ion Transport}

We now consider ion diffusion coefficients. Again, to facilitate comparison with previous results, we express the main ion flux as

$$
\begin{aligned}
\Gamma_{i \Phi}= & \frac{Z_{i} T_{e}}{T_{i}} L_{11, \Phi}^{i i}\left[\left[\frac{T_{i}}{Z_{i} T_{e}}\left[\frac{n_{i}^{\prime}}{n_{i}}-\left(\frac{3}{2}-y_{i \Phi}\right) \frac{T_{i}^{\prime}}{T_{i}}\right]-\frac{T_{Z}}{Z T_{e}}\left[\frac{n_{Z}^{\prime}}{n_{Z}}-\left(\frac{3}{2}-y_{Z \Phi}\right) \frac{T_{Z}^{\prime}}{T_{Z}}\right]\right]\right. \\
& -\frac{m_{e} n_{e}\left\{f_{t \Phi} \nu_{e}^{D}\right\}}{\sum_{k \neq i} m_{k} n_{k}\left\{f_{t \Phi} \nu_{k}^{D}\right\}}\left[\frac{1}{Z_{e}}\left[\frac{n_{e}^{\prime}}{n_{e}}-\left(\frac{3}{2}-y_{e \Phi}\right) \frac{T_{e}^{\prime}}{T_{e}}\right]\right. \\
& \left.\left.-\frac{T_{Z}}{Z T_{e}}\left[\frac{n_{Z}^{\prime}}{n_{Z}}-\left(\frac{3}{2}-y_{Z \Phi}\right) \frac{T_{Z}^{\prime}}{T_{Z}}\right]\right]\right]
\end{aligned}
$$

As expected, in the absence of impurities, the above equation reduces to Eq. (29) with $\left(Z_{i} T_{e} / T_{i}\right) L_{11, \Phi}^{i i}=\left(1 / Z_{i}\right) L_{11, \Phi}^{e e}$ to satisfy ambipolarity. In the presence of impurities, the second term is negligible, and we recover an expression which has the same form as the one obtained by Connor [Ref. 7, Eq. (48)]. The transport enhancement factor $F_{11}^{i i}$ and the quantities $y_{i \Phi}, y_{Z \Phi}$ as a function of $X_{0}$ are shown in Figs. 9, 7, and 10 respectively. The behavior of the ion enhancement factor $F_{11}^{i i}$ is qualitatively similar to that of electron enhancement factors. However, ion enhancement factors depend upon the impurity content as well as the type of impurity species.

The dependence of the ion transport enhancement factor $F_{11}^{i i}$ upon impurity concentration is shown in Fig. 11. In the presence of a poloidal electric field of the order $\epsilon$, 


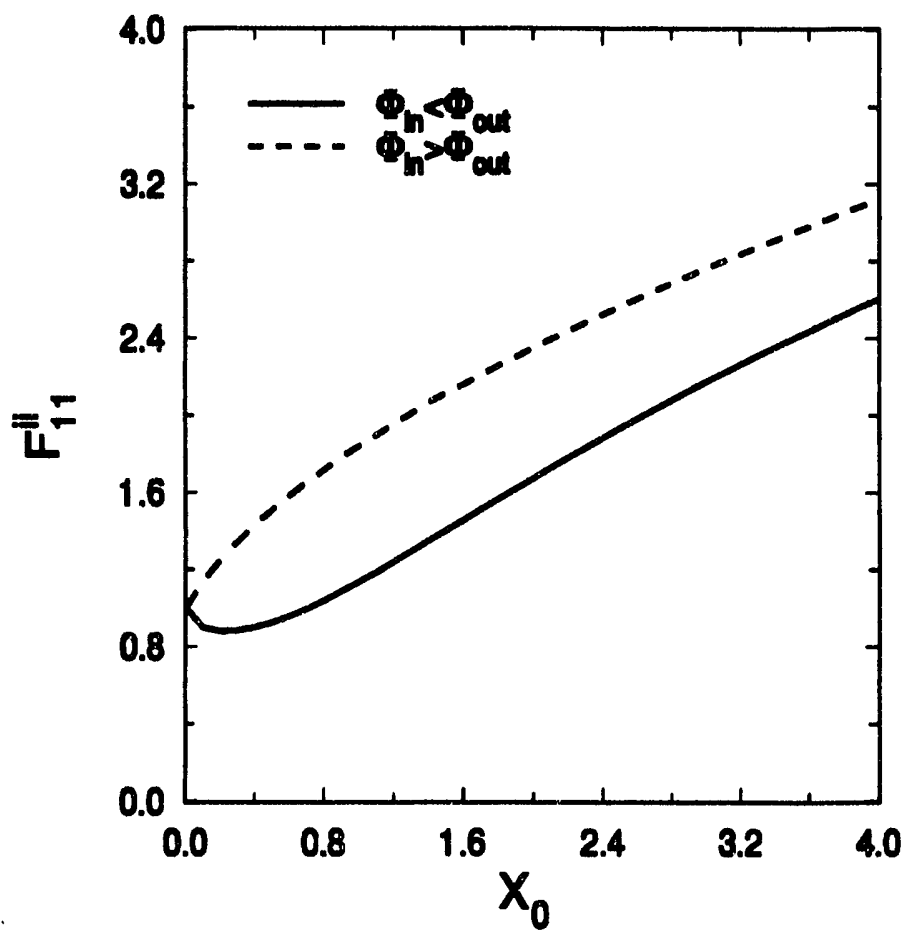

Figure 9: Ion diffusion enhancement factor $F_{11}^{i i}\left(=L_{11, \Phi}^{i i} / L_{11}^{i i}\right)$ as a function of the magnitude of the poloidal potential variation, $X_{0}$ (Impurity: Carbon; $Z_{\text {eff }}=3.0$ ) 


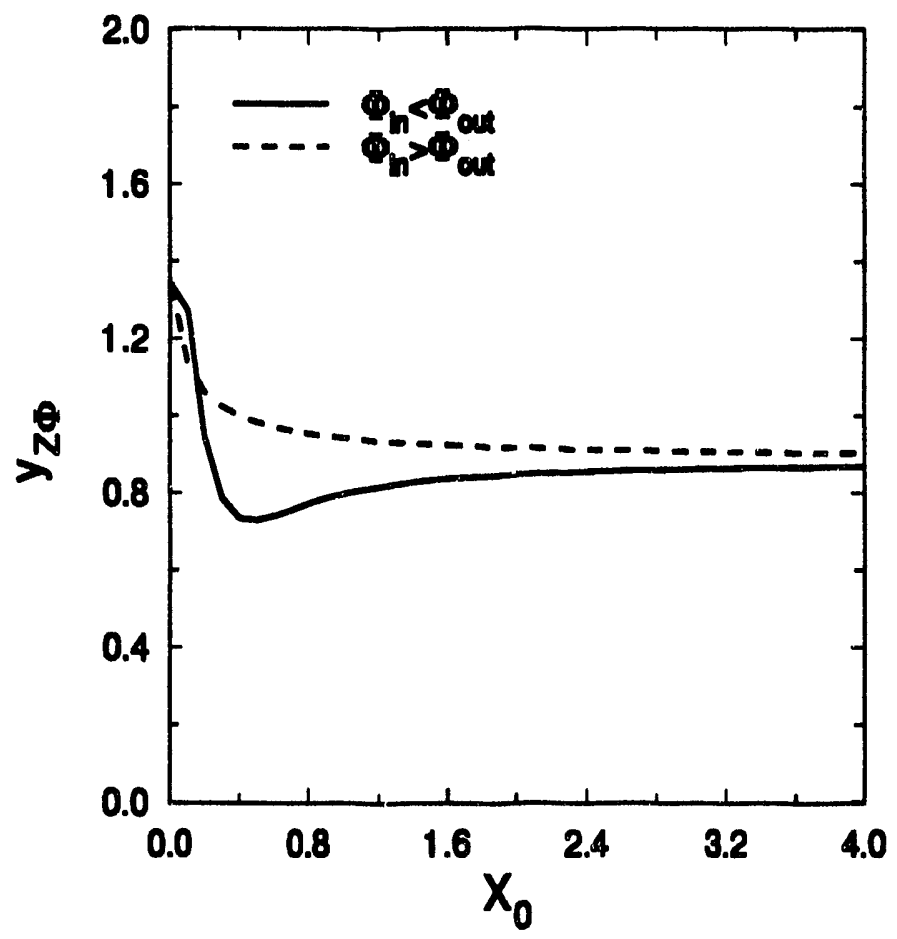

Figure 10: The transport parameter $y_{Z \Phi}$ as a function of $X_{0}$ (Impurity: Carbon; $\left.Z_{\text {eff }}=3.0\right)$ 
i.e. $e \Phi(\theta) / T \sim O(\epsilon)$, due to their larger charge, the fraction of impurity ions trapped in the electrostatic potential well is greater than that of the main ions. This causes a reduction in the collisional coupling between the main ions and the impurity ions, resulting in a drop in the ion transport. This effect is more pronounced for smaller values of $Z_{\text {eff }}$ due to the increasingly important role being played by the impurity species in determining the ion transport.

We now examine the effect of impurity species on transport enhancement. Here, we are primarily interested in the effect of impurity charge and mass upon ion transport at constant $\alpha=n_{Z} Z^{2} / n_{i} Z_{i}^{2}$. The fraction of impurity ions trapped in the electrostatic potential well increases with $Z$. This causes a reduction in the collisional coupling between the impurity ions and the main ions, resulting in a reduction in the ion transport. This effect, which is due to the impurity charge, is somewhat offset by the impurity mass. An increase in the impurity mass leads to an increase in ion transport and it appears that, at least for small to moderate $Z$ impurities, the transport enhancement due to the impurity mass is more important than the transport reduction due to the impurity charge.

Table I shows the transport enhancement factor $F_{11}^{i i}$ in the presence of different impurity species for $\alpha=1$ and $X_{0}=1$. $F_{11}^{i i}$ increases as the impurity mass increases, reaching a maximum for iron. For tungsten, the enhancement factor is smaller than that for iron, apparently indicating the transport reducing effect of impurity charge. We note that the charge state indicated in Table I corresponds to an electron temperature of $\sim 10 \mathrm{keV}$. We also note that a heavy impurity like tungsten may not be in the banana regi ne under conditions that prevail in most tokamaks. The comparison presented in Table I is intended to demonstrate the opposing effects of impurity charge and mass on ion transport enhancement.

Finally, we note that in the absence of a poloidal electric field, the quantities $L_{11, \Phi}, y_{i \Phi}$, and $y_{Z, \Phi}$ depend upon the impurity mass. Our calculations indicate that the discrepancy between our results and those obtained by Connor, ${ }^{7}$ using the heavy impurity assumption, can be quite large when the impurity mass is small. For example, when the impurity strength parameter $\alpha=1$, the discrepancy in $L_{11}^{i i}, y_{i}$, and $y_{z}$ can be $\sim 90 \%, 30 \%$, and $20 \%$ respectively for carbon impurity. The differences 


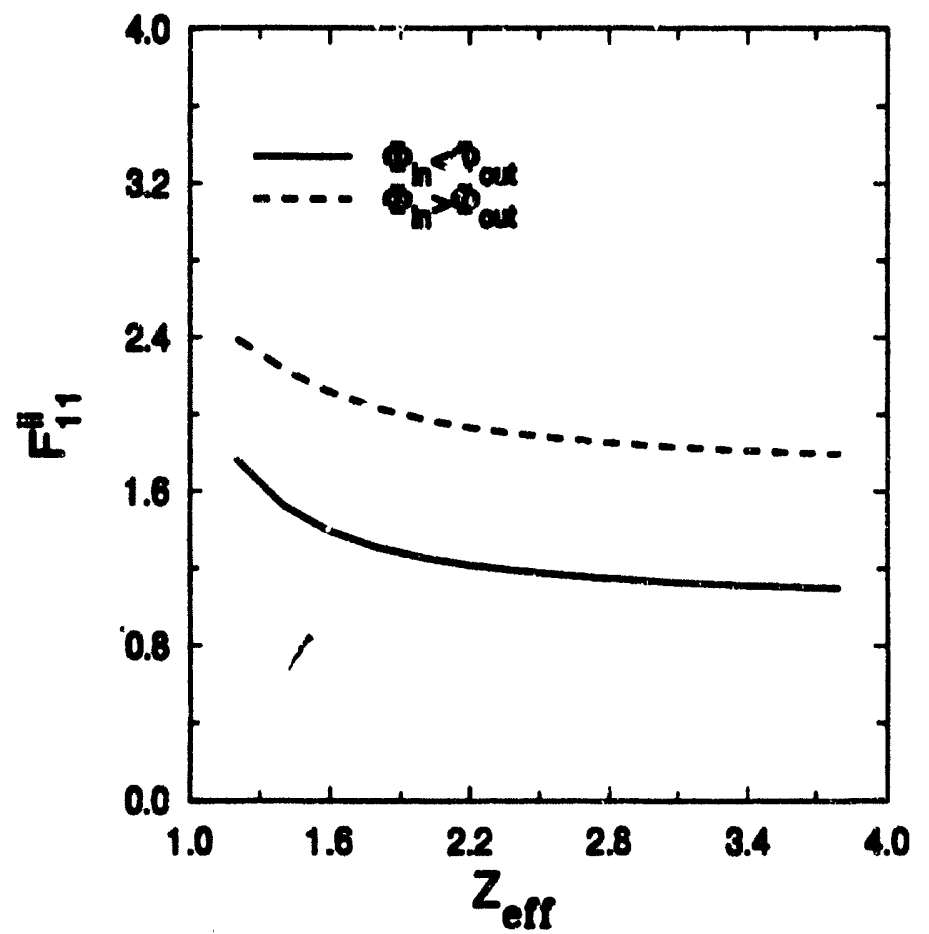

Figure 11: Inn diffusion enhancement factor $F_{11}^{i i}$ as a function of impurity concentration, $Z_{\text {eff }}$ (Impurity: Carbon; $X_{0}=1.0$ ) 
TABLE I: Effect of impurity species on the ion transport enhancement factor $F_{11}^{i i}$ $\left(\alpha=n_{Z} Z^{2} / n_{i} Z_{i}^{2}=1 ; X_{0}=1\right)$.

\begin{tabular}{|l|c|c|c|r|}
\hline \hline \multirow{2}{*}{ Impurity } & \multirow{2}{*}{$Z$} & \multirow{2}{*}{$m_{Z} / m_{i}$} & \multicolumn{2}{|c|}{$F_{11}^{\text {ii }}$} \\
\cline { 4 - 5 } & & & $\Phi_{\text {in }}<\Phi_{\text {out }}$ & $\Phi_{\text {in }}>\Phi_{\text {out }}$ \\
\hline \hline Helium & 2 & 2 & 1.098 & 1.777 \\
\hline Carbon & 6 & 6 & 1.342 & 2.065 \\
\hline Iron & 26 & 28 & 1.454 & 2.249 \\
\hline Tungsten & 60 & 92 & 1.427 & 2.201 \\
\hline \hline
\end{tabular}

decrease as the impurity mass increases. However, even for tungsten, the discrepancy in $L_{11}^{i i}$ is $\sim 30 \%$ when $\alpha=1$.

\section{E. Plasma Rotation Velocity}

While deriving the expressions for the diffusive fluxes, we obtained an expression for the common plasma rotation velocity, $V_{\Phi}$ [Eq. (24)]. Neglecting the contribution of electrons, we write the rotation velocity, for a three species plasma as

$$
V_{\Phi}=\sum_{k ; n=1,2} R_{n, \Phi}^{k} A_{n k}
$$

where the rotation coefficients $R_{n, \Phi}^{k}$ can be easily identified by comparing the above equation with Eq. (24). As in the case of transport coefficients, we define the rotation enhancement factors as

$$
F_{R, n}^{k}=\frac{R_{n, \Phi}^{k}}{R_{n}^{k}}
$$

The rotation enhancement coefficients generally decrease with increasing poloidal potential variation. Figure 12 shows the variation of rotation enhancement factor $F_{R, 1}^{i}$ with $X_{0}$. We observe that for constant gradients, the rotation velocity decreases for most values of $X_{0}$. With increasing $X_{0}$, most of the rotation enhancement factors asymptotically approach a constant value. The drop in the rotation enhancement factors is a result of reduction in the collisional coupling between the main ions and impurity ions caused by enhanced trapping due to the poloidal electric field. 


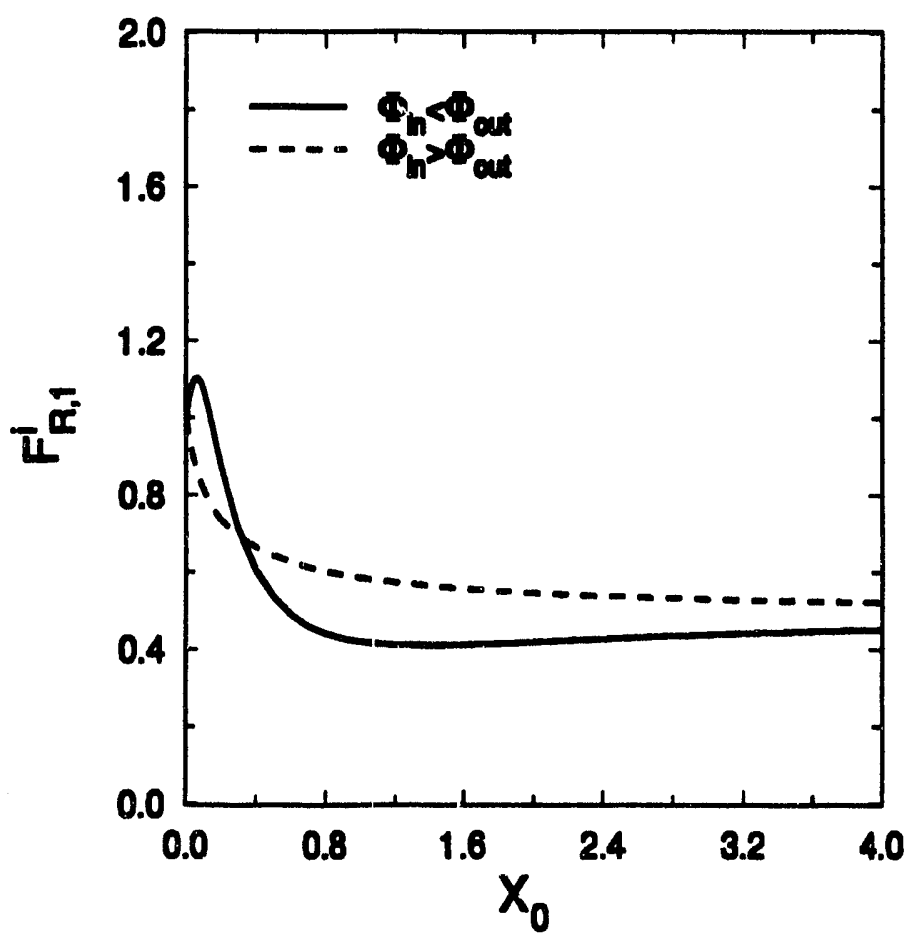

Figure 12: Enhancement of the rotation coefficient $F_{R, 1}^{i}\left(=R_{1, \Phi}^{i} / R_{1}^{i}\right)$ as a function of $X_{0}$ (Impurity: Carbon; $Z_{\text {eff }}=3.0$ ) 
The behavior of the enhancement factors as a function of $Z_{\text {eff }}$ is analogous to the behavior of the ion transport enhancement factor $F_{11}^{i i}$. As with ion transport, the drop in the rotation velocity is caused by increased trapping of the impurity ions due to their larger $Z$. This results in reduced collisional coupling between the main ions and the impurity ions, and hence, reduction in the common flow velocity. Presence of an impurity species of larger $Z$ has a similar effect upon the rotation velocity.

For an electron-ion plasma, Shurygin and Yushmanov ${ }^{3}$ have calculated the radial electric field enhancement factor in the presence of a poloidal electric field. Their calculations indicate that the radial electric field, which is proportional to the rotation velocity, shows a behavior similar to that displayed by the enhancement factors $F_{R, n}^{i}$.

\section{PLASMA CURRENT}

The general expression for the flux surface averaged plasma current is

$$
J=\sum_{j} e_{j} n_{j}<u_{j}>=-\sum_{j} e_{j} n_{j}\left(<u_{e}>-<u_{j}>\right)
$$

where the parallel velocity of the species $j$ is given by

$$
u_{j}=\frac{1}{n_{j}} \int d^{3} v \sigma q f_{j 1}
$$

The procedure used for calculating the parallel velocity is analogous to that used for calculating the restoring coefficients $\left\langle r_{k j}\right\rangle$. Using the solution for $f_{j 1}$, the parallel velocity is found to $\mathrm{be}^{7}$

$$
\begin{aligned}
<u_{j}>= & -\left[\left\{\frac{f_{t \Phi} \nu_{j}^{S}}{\nu_{j \Phi}}\left(\frac{T_{j}}{e_{j} B_{\theta}^{0}}\right) \frac{f_{j 0}^{\prime}}{f_{j 0}}\right\}+\sum_{l}\left\{\frac{f_{t \Phi} \nu_{j l}^{S}}{\nu_{j \Phi}}\right\}<r_{l j}>+\frac{e_{j} A_{3}}{m_{j}}\left\{\frac{f_{t \Phi}}{\nu_{j \Phi}}\right\}\right] \\
& +\left\{\left(\frac{\nu_{j}^{S}}{\nu_{j \Phi}}-1\right)\left(\frac{T_{j}}{e_{j} B_{\theta}^{0}}\right) \frac{f_{j 0}^{\prime}}{f_{j 0}}\right\}+\sum_{l}\left\{\frac{\nu_{j l}^{S}}{\nu_{j \Phi}}\right\}<r_{l j}>+\frac{e_{j} A_{3}}{m_{j}}\left\{\frac{1}{\nu_{j \Phi}}\right\}
\end{aligned}
$$

where

$$
\frac{f_{j 0}^{\prime}}{f_{j 0}}=\left[A_{1 j}+\frac{e_{j}<\Phi>^{\prime}}{T_{j}}+\left(\frac{m_{j} E^{*}}{T_{j}}\right) A_{2 j}\right]
$$

As with particle transport, the current due to the ohmic field and the gradient terms can be calculated by considering the driving forces individually. Again, the radial electric field does not contribute to the current. 


\section{A. Parallel conductivity}

We calculate the parallel conductivity by considering the driving force $A_{3}$. As $\left\langle u_{e}\right\rangle \gg\left\langle u_{i}\right\rangle,\left\langle u_{z}\right\rangle$, we consider only the electron contribution. Furthermore, $\left\langle r_{e e}>\gg<r_{i e}\right\rangle,<r_{Z_{e}}>$ [Eq. (27)]. Using the restoring coefficient $\left.<r_{e e}\right\rangle$ calculated to the order $f_{t \Phi}$ from Eq. (15) in the expression fur $\left\langle u_{e}\right\rangle$, we obtain the ohmic current as

$$
J=\sigma_{\Phi} E_{\|}
$$

where

$$
\sigma_{\Phi}=\frac{n_{e} e^{2}}{m_{e}}\left[\left\{\frac{f_{c \Phi}}{\nu_{e \Phi}}\right\}+\frac{\left\{f_{c \Phi} \nu_{e e}^{S} / \nu_{e \Phi}\right\}^{2}}{\left[\left\{\nu_{e e}^{S}\right\}-\left\{f_{c \Phi} \nu_{e e}^{S} \nu_{e e}^{S} / \nu_{e \Phi}\right\}\right]}\right]
$$

For a large aspect ratio tokamak, we can write $\sigma_{\Phi}$ as

$$
\sigma_{\Phi}=\sigma_{C L}-f_{t} \sigma_{N C, \Phi}
$$

where $\sigma_{C L}$, the conductivity in a plasma with a uniform magnetic field, is given by

$$
\sigma_{C L}=\frac{n_{e} e^{2}}{m_{e}}\left[\frac{1}{\left\{\nu_{e}^{S}\right\}}+\frac{\left\{\nu_{e e}^{S} / \nu_{e}^{S}\right\}^{2}}{\left\{\nu_{e e}^{S}\left(\nu_{e}^{S}-\nu_{e e}^{S}\right) / \nu_{e}^{S}\right\}}\right]
$$

and $\sigma_{N C, \Phi}$, the neoclassical conductivity reduction factor, is given by

$$
\begin{aligned}
\sigma_{N C, \Phi}=\frac{n_{e} e^{2}}{f_{t} m_{e}}\left[\left\{\frac{f_{t \Phi} \nu_{e}^{D}}{\left(\nu_{e}^{S}\right)^{2}}\right\}\right. & +\frac{2\left\{\nu_{e e}^{S} / \nu_{e}^{S}\right\}\left\{f_{t \Phi} \nu_{e e}^{S} \nu_{e}^{D} /\left(\nu_{e}^{S}\right)^{2}\right\}}{\left\{\nu_{e e}^{S}\left(\nu_{e}^{S}-\nu_{e e}^{S}\right) / \nu_{e}^{S}\right\}} \\
+ & \left.\frac{\left\{\nu_{e e}^{S} / \nu_{e}^{S}\right\}^{2}\left\{f_{t \Phi}\left(\nu_{e e}^{S}\right)^{2} \nu_{e}^{D} /\left(\nu_{e}^{S}\right)^{2}\right\}}{\left\{\nu_{e e}^{S}\left(\nu_{e}^{S}-\nu_{e e}^{S}\right) / \nu_{e}^{S}\right\}^{2}}\right]
\end{aligned}
$$

Here, the expression for $\sigma_{N C, \Phi}$ is accurate to order $f_{t}$. When $\tilde{\Phi}(\theta) \simeq 0$, Eq. (37) for the parallel current reduces to Eq. (49) in Ref. 5. Also, when $\nu_{j k}^{S}=\nu_{j k}^{D}$ and $\tilde{\Phi}(\theta)=0$, the expression (37) reduces to Eq. (46) in Ref. 7.

Consistent with our approach in the case of particle transport, we define the enhancement factor for the neoclassical conductivity reduction as

$$
F_{33}=\frac{\sigma_{N C, \Phi}}{\sigma_{N C}}
$$


Figure 13 shows the variation of $F_{33}$ with the magnitude of the poloidal potential variation, $X_{0}$. The behavior of $F_{33}$ is similar to that of the Ware pinch enhancement factor $F_{13}$.

Figure 14 shows the variation of the enhancement factor $F_{33}$ with $Z_{\text {eff }}$. For $\Phi_{i n}>\Phi_{\text {out }}$ with $X_{0}>1$ and for $\Phi_{i n}<\Phi_{\text {out }}$, the enhancement factor $F_{33}$ decreases, signifying a decrease in the neoclassical conductivity reduction factor. However, for $\Phi_{i n}>\Phi_{\text {out }}$ and $X_{0} \sim 1, F_{33}$ increases with $Z_{\text {eff }}$.

Before we attempt an explanation of the behavior of $F_{33}$ with an increase in the impurity content, we note that the neoclassical conductivity reduction factor $\sigma_{N C . \Phi}$ has three terms [see Eq. (41)]. The first term in Eq. (41) contains primarily the effect of electron collisions with main ions and impurity ions, while the other two terms depend mainly on electron-electron collisions. As pointed out by Hirshman et al., ${ }^{4}$ an increase in the impurity content causes a decrease in the neoclassical conductivity reduction by increasing the electron-ion collisions as well as by decreasing the effect of electron-electron collisions. The former effect results in a decrease in the first term in Eq. (41), while the latter effect causes a reduction in the last two terms in Eq. (41).

The presence of a poloidal electric field affects $\sigma_{N C . \Phi}$ primarily by affecting the fraction of trapped particles. When $\Phi_{i n}>\Phi_{\text {out }}$ and $X_{0} \sim 1$, the trapped electron fraction decreases in the region of the velocity space where $v \sim v_{t h}$. Hence, the conductivity reducing effect of impurity ions is weaker, giving rise to a slight increase in $F_{33}$ with $Z_{\text {eff }}$. In all other cases, the fraction of trapped electrons in the region of velocity space where $v \sim v_{t h}$ increases, and hence the conductivity reducing effect of impurity ions is stronger. This results in a decrease in $F_{33}$ with $Z_{\text {eff }}$. We note that the behavior of Ware pinch enhancement factor $F_{13}^{e}$ as a function of $Z_{\text {eff }}$ is similar to that of $F_{33}$. Hence, the present discussion applies to the behavior of $F_{13}^{e}$ as well.

Comparison of the results obtained in this work with those obtained by Chang ${ }^{2}$ in the absence of impurities indicates a $\sim 20 \%$ discrepancy between the two results at $X_{0} \simeq 4$. As with the Ware pinch enhancement factor, the values obtained in the present work are smaller than those obtained Ref. 2. It appears that the discrepancy decreases as $X_{0}$ becomes smaller. We note in passing that in Ref. 2 the analytical 


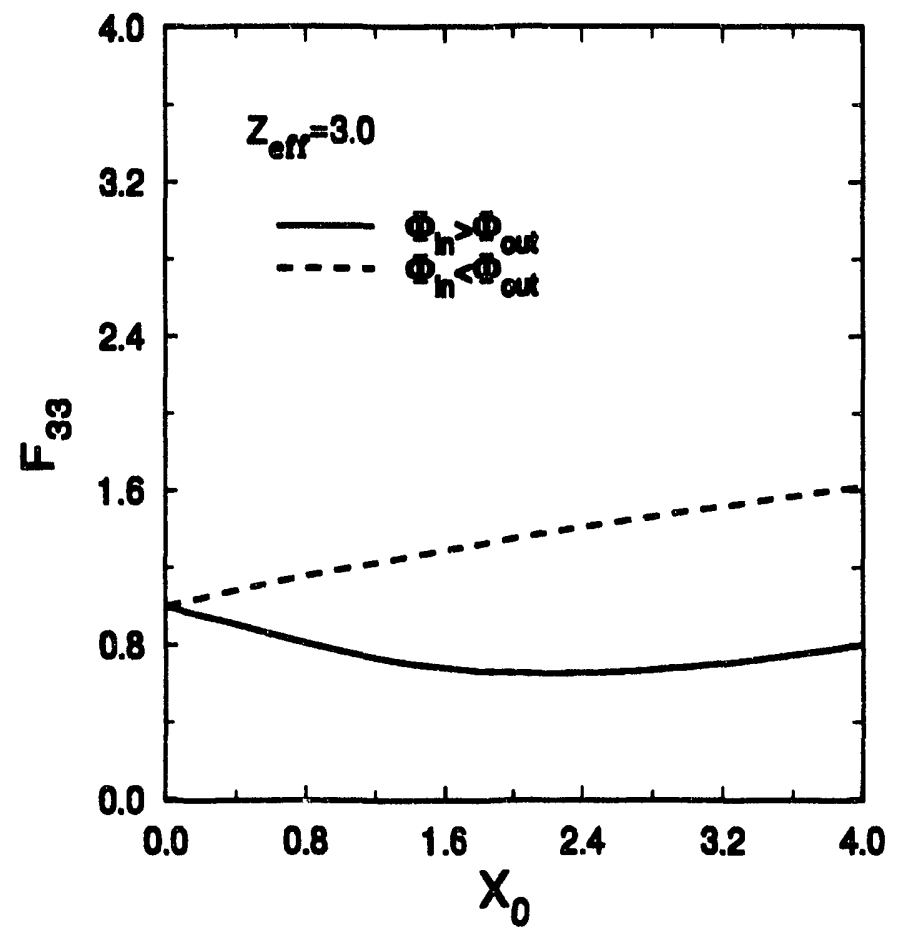

Figure 13: Enhancement $\left(F_{33}\right)$ of the neoclassical conductivity reduction factor as a function of $X_{0}$ 


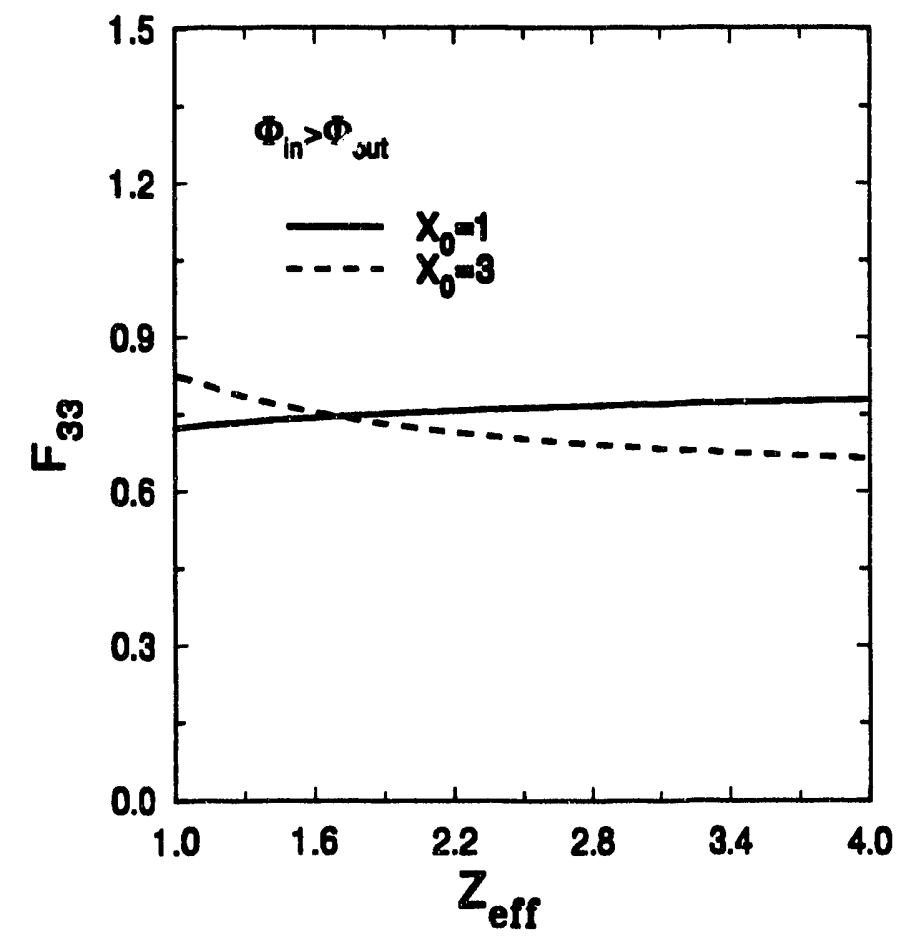

Figure 14: Enhancement $\left(F_{33}\right)$ of the neoclassical conductivity reduction factor as a function of impurity concentration $\left(Z_{\text {eff }}\right)$ for two values of the poloidal potential variation, $X_{0}$ 
fit for the neoclassical conductivity reduction factor $\left(\alpha_{3}, g_{3 e}\right)$ for the case $\Phi_{i n}>\Phi_{\text {out }}$ does not seem to reproduce the curve shown in Fig. 12 of Ref. 2. Our comparison here is with the curve in Fig. 12 in Ref. 2.

Finally, we note that Chang ${ }^{2}$ concludes that the presence of an impurity species in modest amounts $\left(n_{Z} Z \ll n_{e}\right)$ does not alter the transport enhancement factors calculated for an electron-ion plasma. As can be seen from Fig. 14, this clearly is not the case. While the effect of impurities on electron diffusion is insignificant, the effect is significant for the Ware pinch and the conductivity reduction factor, especially at $X_{0}>1$. It appears that the cause of the discrepancy can be found in the way Chang treats the electron collision term. The electron collision term $C_{e}$ can be written as $C_{e}=C_{e e}+C_{e i}+C_{e I}$. If one neglects electron-electron collisions, as Chang seems to do, then $C_{e}=C_{e i}+C_{e I} \simeq\left(\nu_{e i}^{D}+\nu_{e I}^{D}\right) \mathcal{L} \simeq Z_{\text {eff }} \nu_{e i}^{D} \mathcal{L}$, where $\mathcal{L}$ is the Lorentz operator and $Z_{e f f}=\sum_{j \neq e} n_{j} Z_{j}^{2} / n_{e}$. In this case, the effect of including an impurity species is equivalent to replacing $\nu_{e i}^{D}$ by $Z_{e f f} \nu_{e i}^{D}$ in the expressions for enhancement factors for a simple plasma. $Z_{\text {eff }}$ cancels leaving the enhancement factors unaltered. In the present work, the results on diffusion as well as Ware pinch and conductivity were obtained in the presence of electron-electron collision terms. In this case, $C_{e} \simeq\left(z^{\prime} \mathrm{se}+Z_{\text {eff }} \nu_{\text {ei }}^{D}\right) \mathcal{L}$ and $Z_{\text {eff }}$ does not cancel when we compute the enhancement factors.

\section{B. Bootstrap current}

We now compute the parallel currents due to the perpendicular gradients, $A_{1 j}$ and $A_{2 j}$. In order to calculate the bootstrap current in a n-species plasma, we need to calculate the n-1 parallel velocity differences in the presence of the driving terms $A_{1 j}$ and $A_{2 j}$ [see Eq. (35)]. We will follow the procedure used by Hirshman et al. ${ }^{5}$ for calculating the velocity differences. Using the expressions for the restoring coefficients [Eq. (15)], the parallel velocity [Eq. (36)], and the particle flux in the absence of $A_{3}$ [Eq. (22)], we obtain, correct to first order in $f_{t \Phi}$, the following expression for the parallel friction ${ }^{11}$

$$
\begin{aligned}
<R_{j k}>= & -m_{j} n_{j}\left\{\nu_{j k}^{S}\right\}\left[\left(<u_{j}>-<u_{k}>\right)+\left(u_{j k, \Phi}^{0}-u_{k j, \Phi}^{0}\right)\right. \\
& +\sum_{l}\left[\Delta_{l}^{j k}\left[\left(<u_{l}>-<u_{j}>\right)+u_{l j, \Phi}^{0}\right]\right.
\end{aligned}
$$




$$
\left.\left.-\Delta_{l}^{k j}\left[\left(<u_{l}>-<u_{k}>\right)+u_{l k, \Phi}^{0}\right]\right]\right]
$$

where

$$
u_{p q, \Phi}^{0}=\left(\frac{e_{p} B_{\theta}^{0}}{m_{p} n_{p}}\right) \frac{f_{t} \Gamma_{p \Phi}}{\left\{f_{t \Phi} \nu_{p}^{D}\right\}} \gamma_{n \Phi}^{p q}+\left(\frac{T_{p}}{e_{p} B_{\theta}^{01}}\right) f_{t} \gamma_{T \Phi}^{p q} A_{2 p}
$$

and

$$
\begin{aligned}
\gamma_{n \Phi}^{p q}= & \frac{1}{f_{t}}\left[\left\{f_{t \Phi} \frac{\nu_{p q}^{S} \nu_{p}^{D}}{\nu_{p}^{S}}\right\} \frac{1}{\left\{\nu_{p q}^{S}\right\}}-\left\{f_{t \Phi} \frac{\nu_{p}^{D}}{\nu_{p}^{S}}\right\}\right] \\
\gamma_{T \Phi}^{p q}= & \frac{\left\{f_{t \Phi} \nu_{p}^{D}\left(m_{p} E^{*} / T_{p}\right)\right\}}{\left\{f_{t \Phi} \nu_{p}^{D}\right\}} \gamma_{n \Phi}^{p q}-\frac{1}{f_{t}}\left[\left\{f_{t \Phi}\left(\frac{m_{p} E^{*}}{T_{p}}\right) \frac{\nu_{p q}^{S} \nu_{p}^{D}}{\nu_{p}^{S}}\right\} \frac{1}{\left\{\nu_{p q}^{S}\right\}}\right. \\
& \left.-\left\{f_{t \Phi}\left(\frac{m_{p} E^{*}}{T_{p}}\right) \frac{\nu_{p}^{D}}{\nu_{p}^{S}}\right\}\right] \\
\Delta_{l}^{p q}= & {\left[\left\{\frac{\nu_{p q}^{S} \nu_{p l}^{S}}{\nu_{p}^{S}}\right\} \frac{1}{\left\{\nu_{p q}^{S}\right\}}-\left\{\frac{\nu_{p l}^{S}}{\nu_{p}^{S}}\right\}\right] }
\end{aligned}
$$

The above expression for $<R_{j k}>$ is accurate to the order $\Delta_{l}^{p q}\left(\Delta_{l}^{p q}<1\right)$. We have used $\nu_{p \Phi} \simeq \nu_{p}^{S}$ in deriving the above expressions. We note that the quantities $u_{p q, \Phi}^{0}, \gamma_{n \Phi}^{p q}$, and $\gamma_{T \Phi}^{p q}$ have a form similar to those of $u_{p q}^{0}, \gamma_{n}^{p q}$, and $\gamma_{T}^{p q}$ defined by Eqs. (51a), (51c), and (51d) in Ref. 5. The expression for $\Delta_{l}^{p q}$ is identical to that given by Eq. (51b) in Ref. 5. The difference between $u_{p q, \Phi}^{0}, \gamma_{n \Phi}^{p q}$, and $\gamma_{T \Phi}^{p q}$ and the corresponding quantities in Ref. 5 is due to the energy dependence of the quantity $f_{t \Phi}$ when $\tilde{\Phi}(\theta) \neq 0$. Setting $\tilde{\Phi}(\theta)=0$ causes the differences to vanish.

An important point concerns the quantity $\gamma_{n \Phi}^{p q}$. As pointed out by Hirshman et $a l .{ }^{5}$ in the absence of a poloidal potential variation, $\gamma_{n \Phi}^{p q}=\gamma_{n}^{p q} \neq 0$ when $\nu_{p q}^{S} \neq \nu_{p q}^{D}$. In other words, the component of the parallel friction proportional to $\gamma_{n}^{p q}$ is driven by the difference in the slowing down and pitch angle diffusion frequencies. Setting $\nu_{p q}^{S}=\nu_{p q}^{D}$ causes this component of the friction to vanish. In the presence of a poloidal potential variation, setting $\nu_{p q}^{S}=\nu_{p q}^{D}$ does not cause $\gamma_{n \Phi}^{p q}$ to vanish. In this case, the component of the parallel friction proportional to $\gamma_{n \Phi}^{p q}$ is driven by the difference between $\nu_{p q}^{S}$ and $\nu_{p q}^{D}$ as well as the energy dependence of the neoclassical factor $f_{t \Phi}$.

The diffusive particle flux can be written in terms of the parallel friction as

$$
\Gamma_{j \Phi}=-\left\langle\sum_{k} \frac{R_{j k}}{e_{j} B_{\theta}}\right\rangle
$$


Using Eq. (43) for the parallel friction in the above equation for the particle flux, and retaining only the gradient terms, we obtain the following equation for the parallel velocity differences ${ }^{11}$

$$
\sum_{k} m_{j} n_{j} \bar{\nu}_{j k}^{S}\left(<u_{j}>-<u_{k}>\right)=\Gamma_{j \Phi}\left(e_{j} B_{\theta}^{0}\right)+<R_{j 0, \Phi}>
$$

where

$$
\bar{\nu}_{j k}^{S}=\left\{\nu_{j}^{S}\right\}\left\{\frac{\nu_{j k}^{S}}{\nu_{j}^{S}}\right\}+\sum_{l}\left\{\nu_{j l}^{S}\right\} \Delta_{k}^{l j}
$$

and

$$
<R_{j 0, \Phi}>=m_{j} n_{j} \sum_{k}\left[\left\{\nu_{j}^{S}\right\}\left\{\frac{\nu_{j k}^{S}}{\nu_{j}^{S}}\right\} u_{k j, \Phi}^{0}-\left[\left\{\nu_{j k}^{S}\right\} u_{j k, \Phi}^{0}-\sum_{l}\left\{\nu_{j k}^{S}\right\} \Delta_{l}^{k j} u_{l k, \Phi}^{0}\right]\right]
$$

Here, $\bar{\nu}_{j k}^{S}$ is identical to the quantity $\bar{\nu}_{S}^{a b}$ and $\left\langle R_{j 0, \Phi}>\right.$ is similar in its form to $\bar{R}_{a 0}$ in Ref. 5.

Equation (49) provides an expression for the (n-1) parallel velocity differences that can be used in the Eq. (35) to obtain the bootstrap current. Due to the algebraic complexity involved in the calculations, we calculate the bootstrap current for a twoion species plasma. Unlike the calculations in Ref. 5, our calculations are valid for a plasma with an impurity species of arbitrary mass. The expression for the bootstrap current can be written as ${ }^{5}$

$$
J_{n c, \Phi}=\sum_{k ; m=1,2} T_{k} L_{3 m, \Phi}^{k} A_{m k}
$$

where the bootstrap current coefficients are of the form

$$
\begin{aligned}
& L_{31, \Phi}^{k}=L_{0} \frac{C_{e i}}{T_{k}}\left[\hat{C}_{e I, \Phi} L_{11, \Phi}^{e k}+\hat{C}_{i I, \Phi} L_{11, \Phi}^{i k}\right] \\
& L_{32, \Phi}^{k}=L_{0} \frac{C_{e i}}{T_{k}}\left[\hat{C}_{e I, \Phi} L_{12, \Phi}^{e k}+\hat{C}_{i I, \Phi} L_{12, \Phi}^{i k}+\hat{D}_{k \Phi}\right]
\end{aligned}
$$

Here, $L_{1 n, \Phi}^{j k}$ are the diffusion coefficients [see Eq. (25)] and

$$
\begin{gathered}
L_{0}=-f_{t} \frac{n_{e}}{B_{\theta}^{0}} \\
C_{e i}=\frac{n_{i}}{n_{e}} \frac{e Z_{i}}{f_{t}} \frac{B_{\theta}^{0}}{\bar{\nu}_{e i}^{S} \bar{\nu}}
\end{gathered}
$$


The quantities $\hat{C}_{e I, \Phi}, \hat{C}_{i I, \Phi}$, and $\hat{D}_{k, \Phi}$ are complicated functions of the collision frequencies. ${ }^{11}$ Our calculations indicate that

$$
\frac{\hat{C}_{e I, \Phi}}{\hat{C}_{i I, \Phi}} \sim O\left(\sqrt{\frac{m_{i}}{m_{e}}}\right)
$$

From the expressions (53), (54) for the bootstrap current coefficients and the expressions for the diffusion coefficients $L_{11, \Phi}^{j k}$ in Eq. (25), it is easy to see that

$$
L_{31, \Phi}^{e}-Z_{i} L_{31, \Phi}^{i}=Z L_{31, \Phi}^{Z}
$$

As mentioned in Ref. 5, this is a conseçuence of the ambipolarity of the particle fluxes.

Before proceeding with a discussion of the results, we wish to illustrate the relationship between the bootstrap current and the cross field diffusive particle fluxes. Using the expressions for the particle flux [Eq. (25)] in Eq. (52), we can write the bootstrap curreni as

$$
J_{n c, \Phi}=L_{0} C_{e i}\left[\hat{C}_{e I, \Phi} \Gamma_{e \Phi}+\hat{C}_{i I, \Phi} \Gamma_{i \Phi}+\sum_{k} \hat{D}_{k \Phi} A_{2 k}\right]
$$

For simplicity, we consider the case of zero temperature gradients, i.e., $A_{2 k}=0$. In this case, the last term in Eq. (57) vanishes. In the absence of a significant impurity concentration, $\Gamma_{e \Phi} \sim \Gamma_{i \Phi}$ and, as a consequence of Eq. (55), the second term in Eq. (57) is negligible. In the presence of a significant impurity concentration, a similar situation obtains after the siationary state $\left(\Gamma_{e \Phi} \sim \Gamma_{i \Phi}\right)$ is reached. Before the stationary state is reached,

$$
\frac{\Gamma_{e \Phi}}{\Gamma_{i, \Phi}} \sim O\left(\sqrt{\frac{m_{i}}{m_{e}}}\right)
$$

and the second term in Eq. (57) is comparable to the first term. Thus, in the presence of impurities, enhancement of the ion flux due to a poloidal electric field will have a significant effect on the bootstrap current. We note here that an analysis of the relation between the particle fluxes and the bootstrap current similar to the one given here has been carried out by Connor $^{7}$ in the absence of a poloidal electric field.

We note that, of the six transport coefficients $L_{3 n}^{k}(n=1,2 ; k=e, i, Z)$, only five are independent. The coefficient $L_{31}^{Z}$ is dependent on the transport coefficients $L_{31}^{e}$ 
and $L_{31}^{i}$ [see Eq. (56)]. Furthermore, the coefficient $L_{32}^{Z}$ is generally small. Hence, we will primarily concentrate on the behavior of four transport coefficients, $L_{3 n}^{k}$ in $n=$ $1,2 ; k=e, i)$.

We now present the results of our calculations of the effect of a poloidal electric field upon the bootstrap current coefficients. We consider a two ion species plasma in which the impurity is carbon. We define the bootstrap current enhancement factor as

$$
F_{3 m}^{k}=\frac{L_{3 m, \Phi}^{k}}{L_{3 m}^{k}}
$$

where $L_{3 m}^{k}$ are the transport coefficients in the absence of a poloidal electric field. Due to Onsager symmetry, ${ }^{5} L_{31}^{e}=L_{13}^{e}$, and hence, the variation of $F_{31}^{e}$ with $X_{0}$ is identical to that of the Ware pinch enhancement factor, $F_{13}^{e}$. Variation of the bootstrap current enhancement factor $F_{31}^{i}$ as a function of the poloidal electric field is shown in Fig. 15. The variation of the enhancement factors $F_{32}^{k}$ is similar to that of $F_{31}^{k}$. As can be seen from Figs. 8 and 15, the enhancement of the bootstrap current coefficients over their magnitude for $\Phi(\theta)=0$ is at most by a factor of 2. Furthermore, it is clear from Figs. 8 and 15 that the electron and ion enhancement factors $\left(F_{3 n}^{e}\right.$ and $\left.F_{3 n}^{i}\right)$ behave differently for $\Phi_{i n}>\Phi_{\text {out }}$ than for $\Phi_{i n}<\Phi_{\text {out }}$. Hence, the effect of a poloidal electric field upon the bootstrap current cannot be deduced easily from the behavior of the individual enhancement factors. We will evaluate the effect of the enhancement of bootstrap current coefficients and temperature/density profiles upon the magnitude of the bootstrap current later in this section.

Figure 16 shows the variation of the bootstrap current enhancement factor $F_{31}^{i}$ as a function of the impurity concentration $\left(Z_{e f f}\right)$ for $X_{0}=1$. Due to Onsager symmetry, the variation of $F_{31}^{e}$ is identical to that of $F_{13}^{e}$. The variation of $F_{32}^{k}$ with $Z_{\text {eff }}$ is similar to that of $F_{31}^{k}$. As with the diffusion coefficients, the variation of the bootstrap enhancement factors with $Z_{\text {eff }}$ is more pronounced for smaller values of $Z_{\text {eff }}$ due to the increasingiy important role being played by the impurity species in this range of $Z_{\text {eff }}$. As $Z_{\text {eff }}$ increases, the transport enhancement factors asymptotically reach a constant value. The presence of a poloidal electric field in an impure plasma affects the bootstrap current primarily by increasing (or decreasing) the trapped ion or impurity fraction in addition to the trapped electron fraction, and hence the collisional 


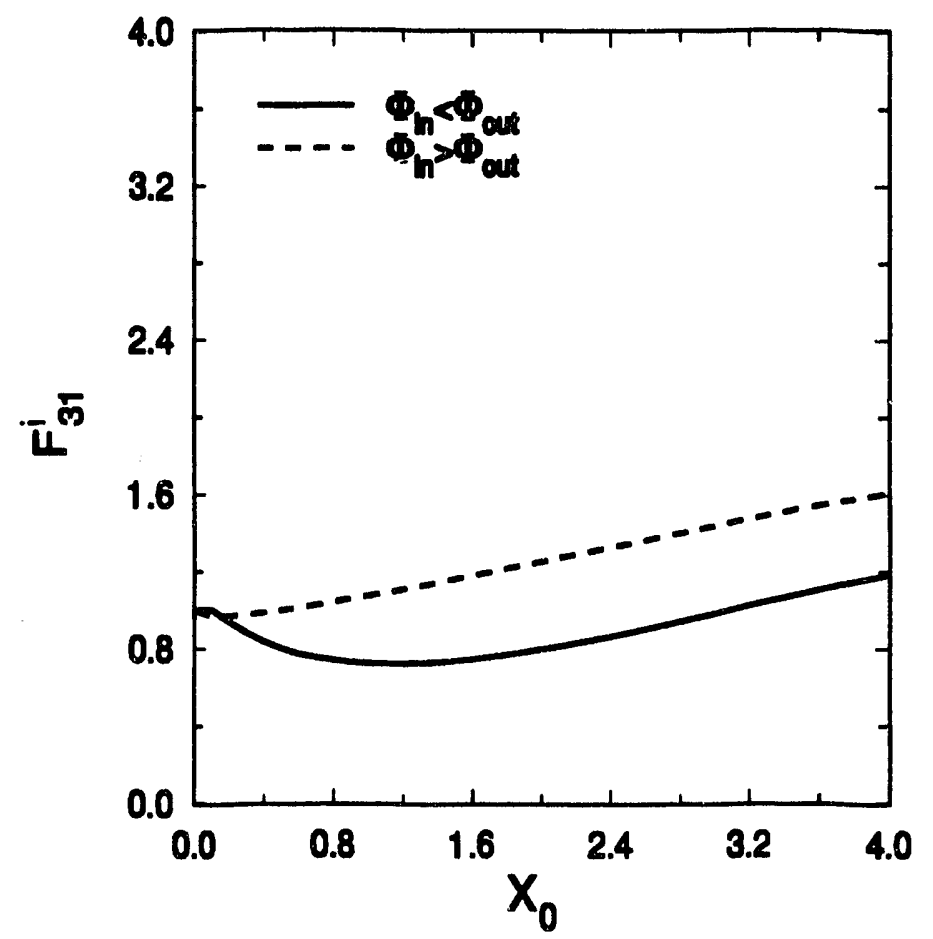

Figure 15: Bootstrap current enhancement factor $F_{31}^{i}$ as a function of, $X_{0}$ (Impurity: Carbon; $Z_{\text {eff }}=3.0$ ) 


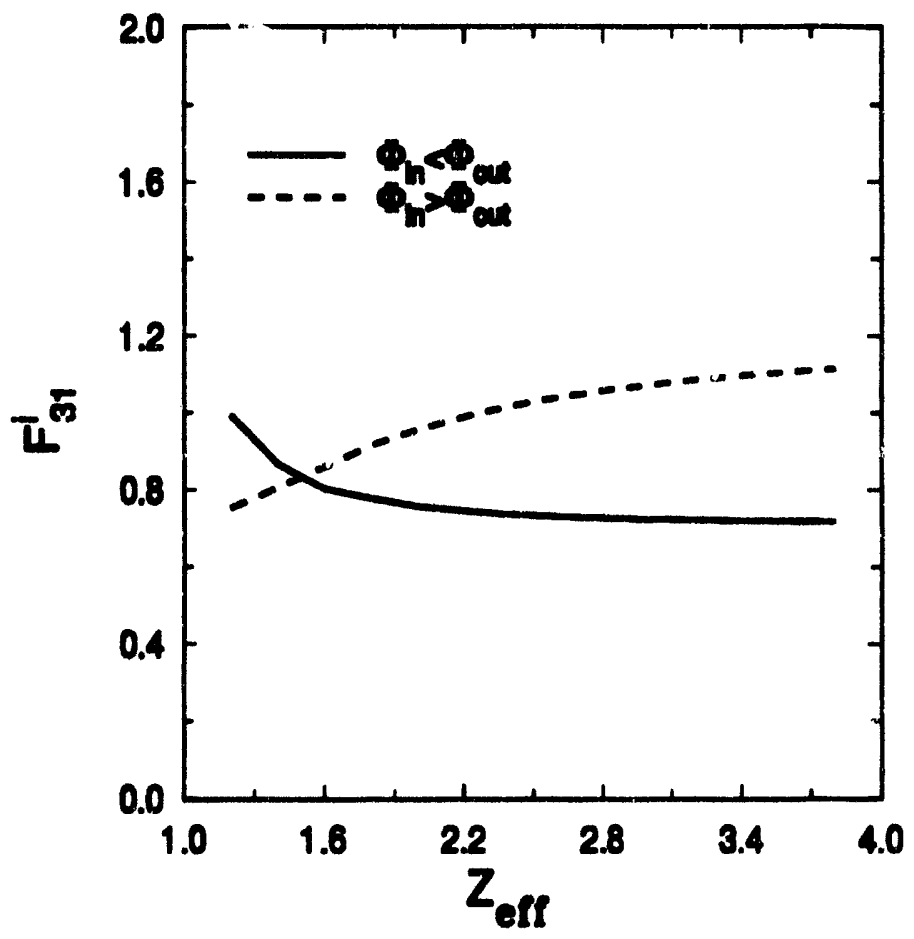

Figure 16: Bootstrap current enhancement factor $F_{31}^{i}$ as a function of impurity concentration, $Z_{\text {eff }}$ (Impurity: Carbon; $X_{0}=1.0$ ) 
coupling between them. We recall here that the bootstrap current is primarily a trapped particle effect. It appears that when $\Phi_{i n}<\Phi_{\text {out }}$, for moderate values of $X_{0}$ $\left(X_{0}<4\right)$, the trapped ion population decreases, resulting in a decrease in $F_{31}^{i}$. For $\Phi_{\text {in }}>\Phi_{\text {out }}$, the opposite effect occurs, i.e., the trapped ion fraction increases, causing an increase in $F_{31}^{i}$ (see Fig. 16).

We now compare our results on the bootstrap current with the results obtained by Chang ${ }^{2}$ in the absence of impurities. In the absence of impurities, Eq. (52) can be written as

$$
\begin{aligned}
J_{n c, \Phi}= & T_{e} L_{31, \Phi}^{e}\left(1+\frac{T_{i}}{T_{e} Z_{i}}\right) \frac{n_{e}^{\prime}}{n_{e}}+T_{e}\left[L_{31, \Phi}^{e}+\left(L_{32, \Phi}^{e}-\frac{5}{2} L_{31, \Phi}^{e}\right)\right] \frac{T_{e}^{\prime}}{T_{e}} \\
& +T_{e} L_{31, \Phi}^{e}\left(\frac{T_{i}}{Z_{i} T_{e}}\right)\left(\frac{Z_{i} L_{32 . \Phi}^{i}}{L_{31, \Phi}^{e}}-\frac{3}{2}\right) \frac{T_{i}^{\prime}}{T_{i}}
\end{aligned}
$$

Using the expressions for the the transport coefficients $L_{32 . \Phi}$ and $L_{31 . \Phi}$, it can be shown that, ${ }^{11}$

$$
\frac{Z_{i} L_{32, \Phi}^{i}}{L_{31, \Phi}^{i}}=\frac{\left\{f_{t \Phi} \nu_{i}^{D}\left(m_{i} E^{*} / T_{i}\right)\right\}}{\left\{f_{t \Phi} \nu_{i}^{D}\right\}}=y_{i \Phi}
$$

With this result, the Eq. (59) has the same form as the expression for the bootstrap current obtained by Chang. ${ }^{2}$ We note that $L_{31, \Phi}$ and $y_{i \Phi}$ defined here are equivalent to $\left(\alpha_{1}, g_{3 e}\right)$ and the variational parameter $y$ defined in Ref. 2. Thus our previous cor:parison of the Ware pinch enhancement factor and the variational parameter in Section IV. giva, the desired comparison between the bootstrap current coefficients.

Finally, we point out that for $\tilde{\Phi}(\theta)=0$, our calculations agree with the transport coefficients calculated using the analytical expressions obtained by Hirshman et al. ${ }^{5,12}$ for a heavy impurity such as iron or tungsten. For lighter impurities, such as carbon or helium, the ion enhancement factors $F_{3 n}^{i}$ differ from those calculated using the expressions in Refs. 5,12 by less than $3 \%$ when the impurity content is low $\left(Z_{\text {eff }}<\right.$ 1.5). For a lighter impurity like carbon, the difference in $F_{3 n}^{i}$ calculated using the two approaches can be as high as $20 \%$ for higher impurity concentrations $\left(Z_{\text {eff }} \simeq\right.$ 3.0). This is to be expected as the expressions in Refs. 5, 12 were obtained in the limit $m_{z} / m_{i} \gg 1$. Nevertheless, the analytic expressions in Refs. 5,12 provide a convenient means for calculating the bootstrap current coefficients for most impurities and concentration if one could tolerate a discrepancy of the order $\sim 20 \%$ for low $\mathrm{Z}$ 
impurities at higher concentrations.

\section{Enhancement of bootstrap current and profile effects}

The bootstrap current depends upon the transport coefficients $L_{3 n}^{k}$ as well as the driving forces $A_{1 k}$ and $A_{2 k}$. As the driving forces depend upon the density and temperature profiles, the enhancement (or reduction) of bootstrap current is dependent on the enhancement (or reduction) of the transport coefficients as well as the electron and ion temperature/density profiles. We consider briefly the profile effects as well as the enhancement of transport coefficients upon the magnitude of the bootstrap current.

We begin by considering a situation where the temperature and density profiles can be approximated by

$$
T_{j}(r)=T_{j 0}\left(1-\rho^{2}\right)^{\alpha_{T}}
$$

and

$$
n_{j}(r)=n_{j 0}\left(1-\rho^{2}\right)^{\alpha_{n j}}
$$

Here $\rho=r / a$ where $a$ is the minor radius. For simplicity, we consider a situation where $T_{e} \simeq T_{i} \simeq T_{Z}$. With this,

$$
\frac{T_{j}^{\prime}}{T_{j}}=\frac{T^{\prime}}{T}=-\frac{2 \alpha_{T}}{a}\left(\frac{\rho}{1-\rho^{2}}\right)
$$

and

$$
\frac{n_{j}^{\prime}}{n_{j}}=-\frac{2 \alpha_{n e}}{a}\left(\frac{\rho}{1-\rho^{2}}\right)
$$

We shall further assume that $Z_{\text {eff }}$ is constant in the core region of the plasma where the present formalism is expected to be valid. This leads to

$$
\frac{n_{j}^{\prime}}{n_{j}}=\frac{n^{\prime}}{n}=-\frac{2 \alpha_{n}}{a}\left(\frac{\rho}{1-\rho^{2}}\right)
$$

With this, Eq. (52) for the bootstrap current can be written as

$$
J_{n c, \Phi}=\frac{2 T}{a} \frac{\rho}{1-\rho^{2}}\left[\alpha_{T} \sum_{k}\left(1.5 L_{31, \Phi}^{k}-L_{32, \Phi}^{k}\right)-\alpha_{n} \sum_{k} L_{31, \Phi}^{k}\right]
$$


We recall that the subscript $\Phi$ indicates the presence of a significant poloidal electric field. In the absence of a poloidal electric field, we have

$$
J_{n c}=\frac{2 T}{a} \frac{\rho}{1-\rho^{2}}\left[\alpha_{T} \sum_{k}\left(1.5 L_{31}^{k}-L_{32}^{k}\right)-\alpha_{n} \sum_{k} L_{31}^{k}\right]
$$

Setting $J_{n c, \Phi}-J_{n c} \geq 0$, we obtain relation between $\alpha_{n}$ and $\alpha_{T}$ for the change in the bootstrap current due to inclusion of a poloidal electric field to be positive. We note that the relationship between $\alpha_{n}$ and $\alpha_{T}$ depends upon the impurity species, concentration, and the sign and magnitude of the potential variation. As an example, we consider the case of carbon impurity with $Z_{\text {eff }}=3.0$. Using the numerically computed transport coefficients, ${ }^{11}$ we find that the condition for an enhancement of the bootstrap current due to a poloidal electric field is

(a)When $\Phi_{\text {in }}<\Phi_{\text {out }}$ :

For $X_{0}=1, \alpha_{n}>0.64 \alpha_{T}$.

For $X_{0}=3, \alpha_{n}>0.49 \alpha_{T}$.

(b) When $\Phi_{\text {in }}>\Phi_{\text {out }}$ :

For $X_{0}=1, \alpha_{n}>-0.36 \alpha_{T}$.

For $X_{0}=3, \alpha_{n}>4.12 \alpha_{T}$.

Figures 17 and 18 show the relation between $\alpha_{n}$ and $\alpha_{T}$, as a function of $X_{0}$, that is needed for an enhancement of the bootstrap current. Under conditions that prevail in most tokamaks, the presence of a poloidal electric field such that $\Phi_{\text {in }}>\Phi_{\text {out }}$ (as during ECRH) results in a reduction in the bootstrap current (Fig. 17). On the other hand, when $\Phi_{\text {in }}<\Phi_{\text {out }}$ (as during ICRH of NBI), the bootstrap current increases if the density profile is more peaked than roughly the square root of the temperature profile, i.e., $\alpha_{n}>0.5 \alpha_{T}$ (see Fig. 18). The behavior of $\alpha_{n} / \alpha_{T}$ depicted in Figs. 17 and 18 follows from the behavior of the enhancement factors $F_{3 n}^{k}$ as a function of $X_{0}$. Finally, we note that varying $Z_{\text {eff }}$ from 2 to 4 has only a minor effect on the behavior of $\alpha_{n} / \alpha_{T}$ shown on Figs. 17 and 18. 


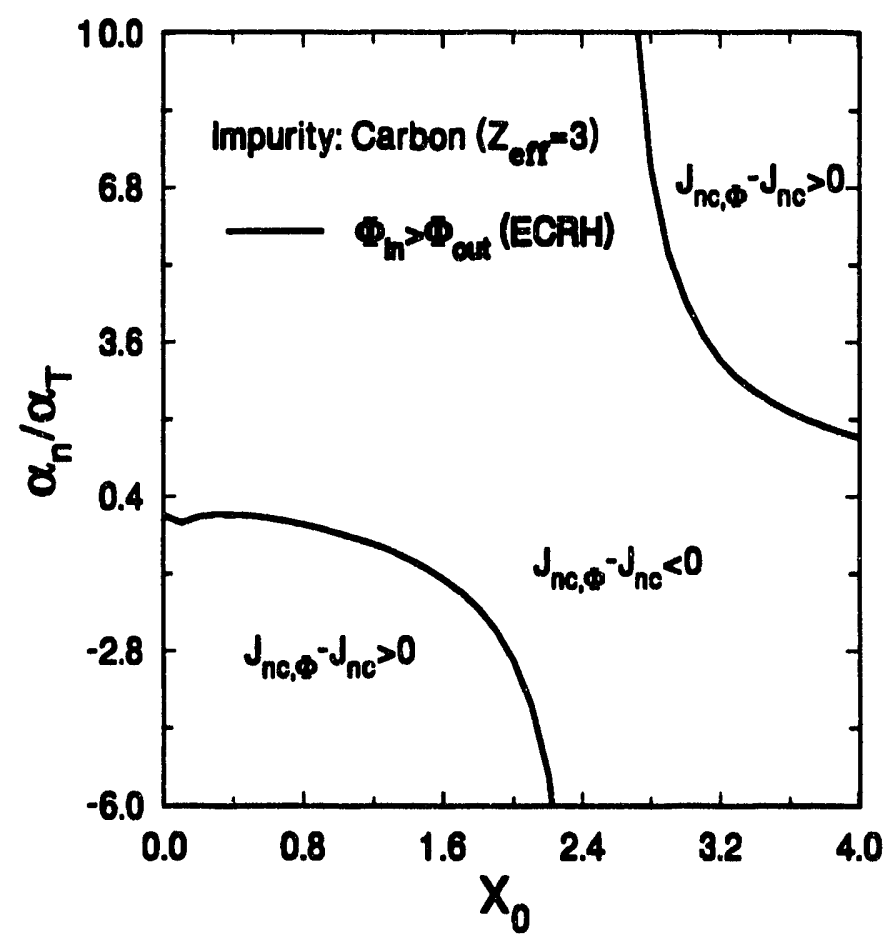

Figure 17: Relation between the density and temperature profiles for the change in the bootstrap current $\left(J_{n c, \Phi}-J_{n c}\right)$ due to the poloidal electric field to be positive when $\Phi_{i n}>\Phi_{\text {out }}$. The profiles are of the form $x_{j}=x_{j}(0)\left[1-(r / a)^{2}\right]^{\alpha}{ }$ where $j=n, T$ 


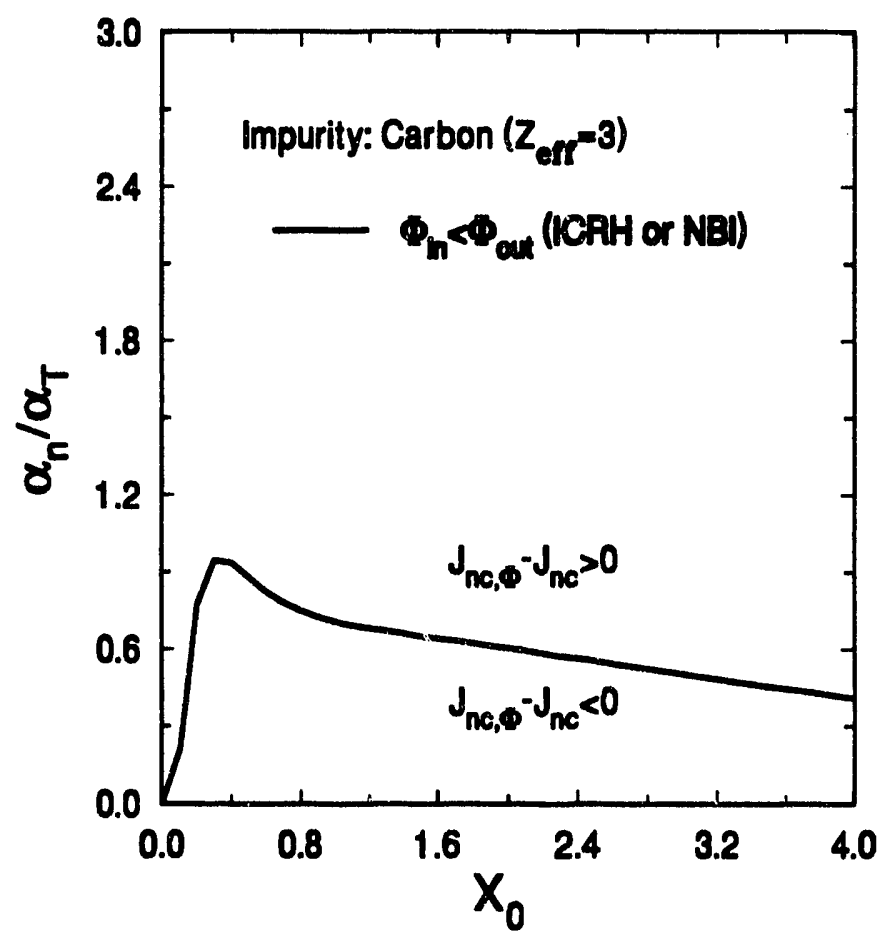

Figure 18: Relation between the density and temperature profiles for the change in the bootstrap current $\left(J_{n c, \Phi}-J_{n c}\right)$ due to the poloidal electric field to be positive when $\Phi_{i n}<\Phi_{\text {out }}$. The profiles are of the form $x_{j}=x_{j}(0)\left[1-(r / a)^{2}\right]^{\alpha_{j}}$ where $j=n, T$. 


\section{SUMMARY AND CONCLUSIONS}

In this paper, we have examined the effect of a poloidal variation in the electrostatic potential of order $\epsilon$ on the neoclassical particle transport and current in a multispecies tokamak plasma. The most significant aspect of the present work is the calculation of the effect of a poloidal electric field on the ion diffusion coefficients, neoclassical conductivity, and bootstrap current.

We find that the main ion diffusion coefficients increase during both ECRH $\left(\Phi_{i_{n}}>\right.$ $\left.\Phi_{\text {out }}\right)$ and ICRH $\left(\Phi_{\text {in }}<\Phi_{\text {out }}\right)$ for most values of the potential variation $\left(X_{0}=\right.$ $e \tilde{\Phi}(\theta) / T \epsilon$ ), implying an increase in both the outward diffusion of main ions and the inward diffusion of impurities. Only when the poloidal potential variation is small, i.e. $X_{0} \leq 1$, do the main ion diffusion coefficients decrease slightly $(\sim 10 \%)$ during ICRH; which implies only a small reduction in the inward impurity diffusion $\left(\Gamma_{Z} \simeq-Z_{i} / Z \Gamma_{i}\right)$. It seems unlikely that any significant reduction in the inward impurity diffusion can be achieved with either ECRH or ICRH. However, variations of this magnitude could be important in interpreting the experimental results on impurity transport, since recent results from $\mathrm{JET}^{13,14}$ indicate that the particle transport in the core region of the plasma is close to the values predicted by the standard $(\tilde{\Phi}(\theta) \simeq 0)$ neoclassical theory. In general, it is expected that modifications of the neoclassical particle transport coefficients of the type examined in this work would be significant in such regimes or regions of the plasma where the particle transport is close to the neoclassical levels.

It is found that in the presence of a poloidal potential variation of order $\epsilon$, the neoclassical conductivity reduction factor $\left(\sigma_{N C}\right)$ decreases by as much as $\sim 35 \%$ (for $\Phi_{\text {in }}>\Phi_{\text {out }}$ ) or increases by $\sim 70 \%$ (for $\Phi_{\text {in }}<\Phi_{\text {out }}$ ) depending on the magnitude of the potential variation. It further appears that the presence of impurities results in a decrease in the neoclassical conductivity reduction factor for most values of the poloidal potential variation. Such variations in the ohmic conductivity can have significant implications for any interpretation of experimental results using a neoclassical conductivity model.

We further find that the presence of a poloidal electric field causes a significant 
change in the bootstrap current coefficients, which can increase by a factor of $\sim 2$ or decrease by $\sim 40 \%$ depending on the magnitude and sign of the potential variation. The change in the bootstrap current, however, depends on the nature of the temperature and density profiles as well as the enhancement (reduction) of the bootstrap current coefficients. We have calculated an increase in the bootstrap current whien $\Phi_{\text {in }}<\Phi_{\text {out }}$ (e.g. ICRH or NBI) if the density profiles are more peaked than roughly the square root of the temperature profiles, $\mathrm{n}$ a deuterium plasma with carbon impurity. It is found that the bootstrap current generally decreases when $\Phi_{i n}>\Phi_{\text {out }}$ (e.g. ECRH). It is important to include the effect of a poloidal electric field in estimating the contribution of the bootstrap current to the total current during strong wave heating or NBI.

\section{ACKNOWLEDGMENT}

This work was supported by the U. S. Department of Energy under Grant No DEFG05-87ER52141. 


\section{APPENDIX A: COLLISION OPERATOR}

The approximate collision operator obtained by Hirshman et al..$^{9,5}$ has the form

$$
C_{j k}\left(f_{j 1}, f_{k 1}\right)=\nu_{j k}^{D} \mathcal{L} f_{j 1}+\frac{2 v_{\|} r_{k j}}{v_{t h . j}^{2}} \nu_{j k}^{S} f_{j 0}+\left[\nu_{j k}^{D}-\nu_{j k}^{S}\right] \frac{v_{\|} u_{j 1}(v)}{v^{2}} f_{j 0}
$$

where

$$
\nu_{j k}^{D}=\nu_{j k}\left[\phi\left(\frac{v}{v_{t h, k}}\right)-G\left(\frac{v}{v_{t h, k}}\right)\right] /\left(\frac{v}{v_{t h, j}}\right)^{3}
$$

is the deflection frequency, ${ }^{5}$

$$
\nu_{j k}^{S}=\frac{2 T_{j 0}}{T_{k 0}}\left(1+\frac{m_{k}}{m_{j}}\right) \nu_{j k} G\left(\frac{v}{v_{t h, k}}\right) /\left(\frac{v}{v_{t h, j}}\right)
$$

is the slowing down frequency. ${ }^{5}$ Here,

$$
\nu_{j k}=4 \pi n_{k} e_{j}^{2} e_{k}^{2} \ln \Lambda /\left[\left(2 T_{j}\right)^{3 / 2} m_{j}^{1 / 2}\right]
$$

where $\ln \Lambda$ is the Coulomb logarithm.

$$
G(x)=\frac{\phi(x)-x \phi^{\prime}(x)}{2 x^{2}}
$$

is the Chandrasekhar function, where $\phi(x)=(2 / \sqrt{\pi}) \int_{0}^{x} \exp \left(-t^{2}\right) d t$ is the error function. The pitch angle operator is defined as

$$
\mathcal{L}=\frac{v_{\|}}{B} \frac{\partial}{\partial \mu}\left(\mu v_{\|}\right) \frac{\partial}{\partial \mu}
$$

Also,

$$
u_{j 1}(v) f_{j 0}=(3 / 4 \pi) \int v_{\|} f_{j 1} d \Omega
$$

where $\left.d \Omega=2 \pi v^{-1} d v_{\|}=\pi \sum_{\sigma}(B d \mu / w)\left(v /\left|v_{\|}\right|\right)\right)^{5,12}$ The momentum restoring coeffcient $r_{k j}$ is defined as

$$
r_{k j}=\frac{\int m_{k} \nu_{k j}^{S} v_{\|} f_{k 1} d^{3} v}{m_{j} n_{j}\left\{\nu_{j k}^{S}\right\}}
$$

where the integration operator \{\} is given by

$$
\begin{aligned}
\left\{F^{j k}(v)\right\} & =2 \int\left(\frac{v_{\|}}{v_{t h j}}\right)^{2} F^{j k}(v) \frac{f_{j 0}}{n_{j}} d^{3} v \\
& =\left(\frac{8}{3 \sqrt{\pi}}\right) \int_{0}^{\infty} x_{j}^{4} \exp \left(-x_{j}^{2}\right) F^{j k}\left(x_{j}\right) d x_{j}
\end{aligned}
$$

Here, $F^{j k}(v)$ is an arbitrary function of the velocity $v$. 


\section{APPENDIX B: CALCULATION OF $f_{t \Phi}$}

The factor $f_{t \Phi}$ is closely related to the fraction of trapped particles. In the absence of poloidal potential variation, the factor has been computed for a large aspect ratio tokamak by Rosenbluth et al. ${ }^{10}$ and more recently by Balescu. ${ }^{15}$ The value of $f_{t \Phi}$ in the absence of a poloidal electric field is approximately $1.46 \sqrt{\epsilon}{ }^{10,15}$ In the presence of poloidal asymmetries in the potential, the trapped particle distribution changes and, as discussed in Section II., the boundary between the trapped-untrapped regions is a function of the particle energy. We will calculate the value of $f_{t \Phi}$ for the case of a large aspect ratio tokamak using a method employed by Chang. ${ }^{2}$ The quantity $f_{t \Phi}$ is given $b^{7,5}$

$$
f_{t \Phi}=\left\langle\sum_{\sigma} \frac{3 B}{4 w} \sigma \frac{v_{t h, j}^{2}}{v} \int d \mu \mu\left[\frac{1}{v_{\|}}-\frac{1}{\left\langle v_{\|}\right\rangle}\right]\right\rangle\left(\frac{B^{0}}{v_{t h, j}^{2}}\right)
$$

Defining $\xi=\left|v_{\|}\right| / v$ and $\lambda^{\prime}=\mu B / w$, we obtain,

$$
\begin{aligned}
f_{t \Phi} & =\sum_{\sigma}\left(\frac{3}{8}\right) \sigma^{2}\left\langle\int d \lambda^{\prime} \lambda^{\prime}\left[\frac{1}{\xi}-\frac{1}{<\xi>}\right]\right\rangle \\
& =\sum_{\sigma}\left(\frac{3}{8}\right) \sigma^{2}\left\langle\int_{T} \frac{d \lambda^{\prime} \lambda^{\prime}}{\xi}\right\rangle+\sum_{\sigma}\left(\frac{3}{8}\right) \sigma^{2}\left\langle\int_{U T} d \lambda^{\prime} \lambda^{\prime}\left[\frac{1}{\xi}-\frac{1}{<\xi>}\right]\right\rangle
\end{aligned}
$$

where the first integral is over the trapped particle region and the second integral is over the untrapped particle region. Here, due to the large aspect ratio assumption, we have used $B \simeq B^{0}$. We now observe that due to the large aspect ratio assumption, and due to our neglect of the boundary layer,

$$
\frac{1}{\xi}-\frac{1}{<\xi>} \sim O(\epsilon)
$$

Furthermore, referring to Figs. 2 and $4, \lambda_{\max }^{\prime} \simeq 1-\epsilon$ over most of the velocity space. Hence the second integral makes a contribution of the order $(1-\epsilon)^{2} O(\epsilon) \sim O(\epsilon)$. Hence, to the lowest order in $\epsilon$, we write

$$
f_{t \Phi}=\sum_{\sigma}\left(\frac{3}{8}\right) \sigma^{2}\left\langle\int_{\lambda_{\min }^{\prime}}^{\lambda_{\max }^{\prime}} \frac{d \lambda^{\prime} \lambda^{\prime}}{\xi}\right\rangle+O(\epsilon)
$$

Expressing $\lambda^{\prime}$ in terms of $\xi$, we obtain

$$
f_{t \Phi}=\sum_{\sigma}\left(\frac{3}{8}\right) \sigma^{2}\left\langle-2 \int_{T}\left(1-\xi^{2}\right) d \xi\right\rangle
$$

We now examine the integration limits. 
1. When $e_{j} \Phi_{\text {in }}<e_{j} \Phi_{\text {out }}$ :

For B-trapping, in terms of the variable $\xi$. [see Eq. (1)],

$$
c_{\text {crit }}=\sqrt{\epsilon}(1+\cos \theta)^{1 / 2}\left[1-\frac{\left|Z_{j}\right| X_{0} T}{m_{j} w}\right]^{1 / 2} \geq \xi \geq 0
$$

For E-trapping, using Eq. (2),

$$
\xi_{\text {crit }}=\sqrt{\epsilon}(1-\cos \theta)^{1 / 2}\left[\frac{\left|Z_{j}\right| X_{0} T}{m_{j} w}-1\right]^{1 / 2} \geq \xi \geq 0
$$

2. When $e_{j} \Phi_{\text {in }}>e_{j} \Phi_{\text {out }}$ :

In this case, we have only B-trapping. From Eq. (5), we get

$$
\xi_{\text {crit }}=\sqrt{\epsilon}(1+\cos \theta)^{1 / 2}\left[1+\frac{\left|Z_{j}\right| X_{0} T}{m_{j} w}\right]^{1 / 2}: \xi \xi \geq 0
$$

From Eqs. (B2) and (B3), we can determine the lower limit on $x_{j}^{2}=m_{j} w / T$ by setting $\xi_{\text {crit }}=1$. This gives,

For $e_{j} \Phi_{i n}<e_{j} \Phi_{\text {out }}$,

$$
x_{j}^{2} \geq \frac{\epsilon(1-\cos \theta)\left|Z_{j}\right| X_{0}}{1+\epsilon(1-\cos \theta)}=x_{j, c r i t}^{2}
$$

For $e_{j} \Phi_{i n}>e_{j} \Phi_{\text {out }}$

$$
x_{j}^{2} \geq \frac{\epsilon(1+\cos \theta)\left|Z_{j}\right| X_{0}}{1-\epsilon(1+\cos \theta)}=x_{j, \text { crit }}^{2}
$$

Using Eqs. (B1), (B2), and (B3), we obtain

$$
f_{t \Phi}=\sum_{\sigma}\left(\frac{3}{4}\right) \sigma^{2}\left\langle\int_{0}^{\xi_{c r i t}}\left(1-\xi^{2}\right) d \xi\right\rangle=\frac{3}{2}\left\langle\xi_{\text {crit }}-\frac{\xi_{\text {crit }}^{3}}{3}\right\rangle
$$

Using Eqs. (B4) and (B5), the maximum value of $f_{t \Phi}$ is found to be 1 . Hence, $f_{t \Phi} \leq 1$. For $x_{j}>x_{j, c r i t}$, we can simplify the above equation by retaining only the first term. In this case,

$$
f_{t \Phi} \simeq \frac{3}{2}<\xi_{\text {crit }}>+O\left(\epsilon^{3 / 2}\right)
$$

Here, we have used $\sum_{\sigma} \sigma^{2}=2$. In the present work, the factor $f_{t \Phi}$ appears in the velocity integrals of the type \{\} [Eq. (A9)]. As will be shown in Appendix C, the contribution to the integral from the low velocity region is negligible for a large aspect 
ratio tokamak. Hence, the above expression for $f_{t \Phi}$ is adequate for our purposes. Using the results (B1), (B2), and (B3), we have for $e_{j} \Phi_{i n}<e_{j} \Phi_{\text {out }}$,

$$
\begin{aligned}
f_{t \Phi} & \simeq \frac{3}{2} \sqrt{\epsilon}\left\langle(1-\cos \theta)^{1 / 2}\right\rangle\left|1-\frac{\left|Z_{j}\right| X_{0} T}{m_{j} w}\right|^{1 / 2} \\
& =f_{t}\left|1-\frac{\left|Z_{j}\right| X_{0} T}{m_{j} w}\right|^{1 / 2}
\end{aligned}
$$

Similarly, for $e_{j} \Phi_{\text {in }}>e_{j} \Phi_{\text {out }}$,

$$
\begin{aligned}
f_{t \Phi} & \simeq \frac{3}{2} \sqrt{\epsilon}\left\langle(1+\cos \theta)^{1 / 2}\right\rangle\left|1+\frac{\left|Z_{j}\right| X_{0} T}{m_{j} w}\right|^{1 / 2} \\
& =f_{t}\left|1+\frac{\left|Z_{j}\right| X_{0} T}{m_{j} w}\right|^{1 / 2}
\end{aligned}
$$

Here, we have used the fact that $\left\langle(1-\cos \theta)^{1 / 2}\right\rangle=\left\langle(1+\cos \theta)^{1 / 2}\right\rangle$. In either case, we note that $f_{t \Phi}>\sqrt{\epsilon}$. This justifies our neglect of the the untrapped particle contribution, which is of the order $\epsilon$. We observe that when $\tilde{\Phi}(\theta) \simeq 0$ i.e., $X_{0} \simeq 0$, we have $f_{t \Phi}=f_{t}=3 / 2<(1 \pm \cos \theta)^{1 / 2}>\sqrt{\epsilon}=1.35 \sqrt{\epsilon}$. This value is somewhat smaller than the value $(1.46 \sqrt{\epsilon})$ quoted before. The main effect of the poloidal electric field is contained in the term $\left|1 \pm \frac{\left|Z_{j}\right| X_{0} T}{m_{j} w}\right|^{1 / 2}$, and hence, for the present wor $\Delta$, the above expressions for $f_{t \Phi}$ are adequate.

\section{APPENDIX C: SIMPLIFICATION OF $\nu_{j \Phi}$}

In the presence of a poloidal electric field, $\nu_{j \Phi}=\left[\nu_{j}^{S}+f_{t \Phi}\left(\nu_{j}^{D}-\nu_{j}^{S}\right)\right]$. Noting that $f_{t \Phi}=$ $f_{t}|1 \pm| Z_{j}\left|X_{0} / x_{j}^{2}\right|^{1 / 2}$, we observe that in the low energy region where $x=v / v_{t h} \ll 1$, $f_{t \Phi}$ can be quite large, approaching 1 . Hence, we have to examine the consequences of $f_{t \Phi} \simeq 1$ in the low energy region on the velocity integrals appearing in the transport coefficients.

We begin by observing that most of the velocity integrals appearing in the transport coefficients are of the type $\left\{f_{t \Phi} F(v) / \nu_{j \Phi}\right\}$ where $F(v)$ is an arbitrary function of velocity. We specifically consider $F(v)=\nu_{j}^{D} \nu_{j}^{S}$. The conclusions drawn below are expected to be valid for other forms of $F(v)$ appearing in the transport cuefficients. 
We have

$$
\begin{aligned}
\left\{\frac{f_{i} F(v)}{\nu_{j \Phi}}\right\} & =\left\{\frac{\nu_{j}^{S} \nu_{j}^{D} f_{t \Phi}}{\nu_{j}^{S}\left[1+f_{t \Phi}\left(\nu_{j}^{D} / \nu_{j}^{S}-1\right)\right]}\right\} \\
& =\frac{8}{3 \sqrt{\pi}} \int_{x_{j, \text { crit }}}^{\infty} x_{j}^{4} \exp \left(-x_{j}^{2}\right)\left[\frac{\nu_{j}^{D} f_{t \Phi}}{1+f_{t \Phi}\left(\nu_{j}^{D} / \nu_{j}^{S}-1\right)}\right] d x_{j}
\end{aligned}
$$

In the absence of a poloidal electric field, $x_{j, c r i t}=0$, and the lower limit in the above integral is zero. In the presence of a poloidal electric field, for large values of $x_{j}$, i.e. $x_{j} \gg 1, f_{t \Phi} \rightarrow f_{t}$ and we can set $\nu_{j \Phi}=\nu_{j}^{S}$. We, therefore, examine only the region of the velocity space where $f_{t \Phi} \simeq 1$. We have, in this region, $x_{j} \sim \mathcal{O}\left(f_{t} \sqrt{X_{0}\left|Z_{j}\right|}\right)<1$ for $f_{t} \rightarrow 0$. Setting $f_{t \Phi}=1$, the integrand in the above equation reduces to

$$
\text { Integrand } \simeq x_{j}^{4} \exp \left(-x_{j}^{2}\right) \nu_{j}^{S}
$$

In this region, $x_{j} \sim \mathcal{O}\left(x_{j, c r i t}\right) \ll 1$, and $\nu_{j}^{S}$ scales as $\sim 1 / x_{j}^{3}$ for electrons and $\sim 1 / x_{j}$ for ion as well as impurity. Hence, the value of the integrand is $\leq x_{j} \exp \left(-x_{j}^{2}\right) \ll 1$, leading to negligible contribution from this region of the velocity space. Hence, the contribution to the velocity integral from the region where $f_{t \Phi} \simeq 1$ is small for a large aspect ratio tokamak. We, therefore, set $\nu_{j \Phi} \simeq \nu_{j}^{S}$ in the transport coefficients. 


\section{REFERENCES}

${ }^{1}$ J. Y. Hsu, V. S. Chan, R. W. Harvey, R. Prater, and S. K. Wong, Phys. Rev. Lett. 53, 564 (1984).

${ }^{2}$ C. S. Chang, Phys. Fluids 26, 2140 (1983).

${ }^{3}$ R. V. Shurygin and P. N. Yushmanov, Sov. J. Plasma. Phys. 12, 306 (1986).

${ }^{4}$ S. P. Hirshman and D. J. Sigmar, Nucl. Fusion 21, 1079 (1981).

${ }^{5}$ S. P. Hirshman, D. J. Sigmar, and J. F. Clarke, Phys. Fluids 19, 656 (1976).

${ }^{6}$ F. L. Hinton and R. D. Hazeltine, Rev. Mod. Phys. 48, 239 (1976).

${ }^{7}$ J. W. Connor, Plasma Phys. 15, 765 (1973).

${ }^{8}$ A. Baños, Jr., Plasma Phys. 1, 305 (1967).

${ }^{9}$ S. P. Hirshman and D. J. Sigmar, Phys. Fluids 19, 1532 (1976).

${ }^{10}$ M. N. Rosenbluth, R. D. Hazeltine, and F. L. Hinton, Phys. Fluids 15, 116 (1972).

${ }^{11} \mathrm{~K}$. Indireshkumar, Ph.D. Thesis, Georgia Insititute of Technology, 1992.

${ }^{12}$ S. P. Hirshman and D. J. Sigmar, Phys. Fluids 20, 418 (1977).

${ }^{13}$ R. Giannella, N. C. Hawkes, L. Lauro Taroni, M. Mattioli, J. O'Rourke, and D. Pasini, Plasma Phys. Contr. Fusion 34, 687 (1992).

${ }^{14}$ D. Pasini, R. Giannella, L. Lauro Taroni, M. Mattioli, B. Denne-Hinnov, N. Hawkes, G. Magyar, and H. Weisen, Plasma Phys. Contr. Fusion 34, 677 (1992).

${ }^{15}$ R. Balescu, Transport Processes in Plasmas, (Elsevier Science Publishers. B. V., Amsterdam, 1988). 

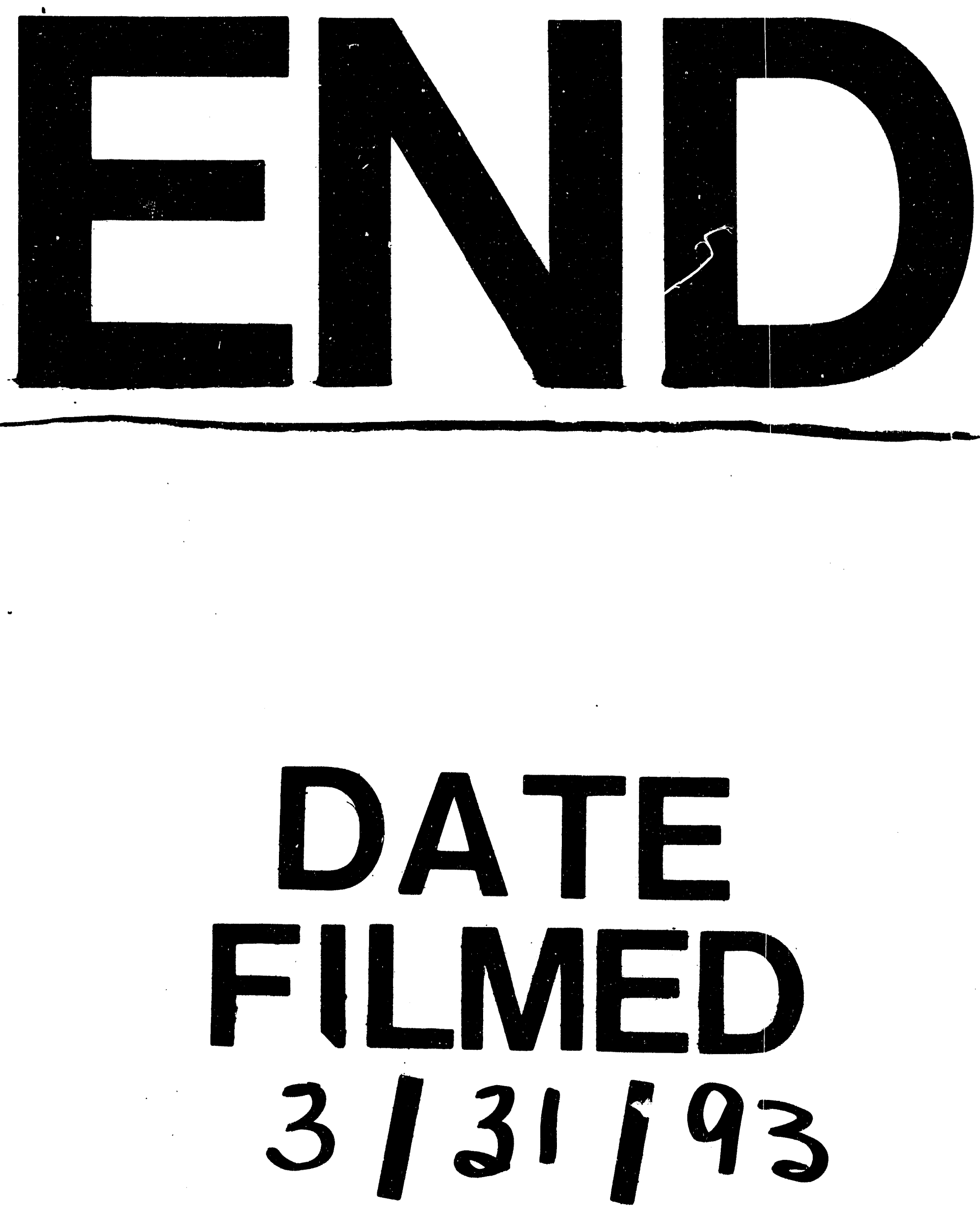
